\title{
A Feedforward Convolutional Neural Network with a Few Million Neurons Learns from Images to Covertly Attend to Cues and Context like Humans and an Optimal Bayesian Observer
}

Sudhanshu Srivastava ${ }^{1,5}$, William Wang ${ }^{3,5}$, Miguel P. Eckstein ${ }^{1,2,4,5}$

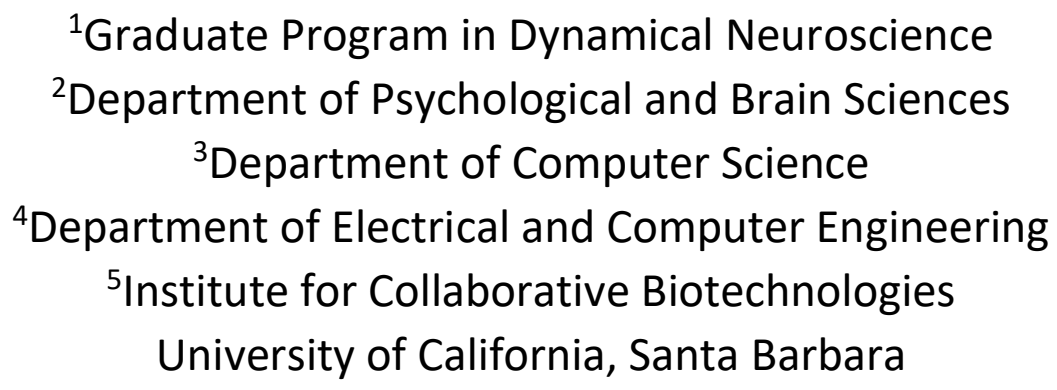

sudhanshu@ucsb.edu,william@cs.ucsb.edu, miguel.eckstein@psych.ucsb.edu 


\section{Abstract}

Human behavioral experiments have led to influential conceptualizations of visual attention, such as a serial processor or a limited resource spotlight. There is growing evidence that simpler organisms such as insects show behavioral signatures associated with human attention. Can those organisms learn such capabilities without conceptualizations of human attention? We show that a feedforward convolutional neural network (CNN) with a few million neurons trained on noisy images to detect targets learns to utilize predictive cues and context. We demonstrate that the CNN predicts human performance and gives rise to the three most prominent behavioral signatures of covert attention: Posner cueing, set-size effects in search, and contextual cueing. The CNN also approximates an ideal Bayesian observer that has all prior knowledge about the statistical properties of the noise, targets, cues, and context. The results help understand how even simple biological organisms show human-like visual attention by implementing neurobiologically plausible simple computations. 


\section{Introduction}

Covert visual attention refers to an observer's ability to select a region or object of a scene without moving their eyes. In the last three decades, three prominent paradigms have been used to experimentally operationalize visual attention and assess its impact on perceptual performance (Figure 1): the Posner cueing task ${ }^{1-3}$, the set-size visual search paradigm ${ }^{4-7}$, and the contextual cueing paradigm ${ }^{8-10}$. These three paradigms have shaped the scientific and classroom conceptualizations of covert attention, including a spotlight metaphor ${ }^{2,11}$, a resource-limited mechanism ${ }^{12,13}$, and a temporally serial processor ${ }^{4,5,14,15}$.

In the cueing paradigm, a cue such as a central arrow or a box indicates with some validity (e.g., $80 \%$ ) the probable location of a target (Figure 1, left). Observers typically find the target faster or more accurately when the cue correctly indicates the target location (valid cue trials) than when it does not (invalid cue trials). The performance advantage on valid-cue trials is attributed to a spotlight or zoom of attention moving to the cued location and enhancing the processing of the target ${ }^{2,11,16}$.

In the visual search paradigm, observers find a target that might be present among distractors (Figure 1, center). The number of distractors (set-size) is manipulated by using cues such as boxes or arrows indicating the locations that the target might appear. As the number of cued locations diminishes, the accuracy of finding the target improves. This improvement is typically interpreted to arise from better guidance of an attentional processor that is serially deployed across candidate cued locations ${ }^{4-7}$

In the contextual cueing task, a tilted T appears among rotated Ls, and the observer decides whether the $\mathrm{T}$ is tilted to the right or left (Figure 1c, right). Unknown to the observer, a subset of configurations and target locations are repeated multiple times while the tilt of the target T varies randomly left or right. Observers typically show improved target-tilt identification for old configurations when compared to novel configurations. Here, the standard explanation is that the implicit recognition of the old background helps allocate attentional resources at the target location and facilitates performance ${ }^{17,18}$.

Initially, the majority of attention studies were conducted with humans. ${ }^{1,2,4,19}$ One line of thinking was of a tight relationship between attention and conscious experience ${ }^{20,21}$ raising the question of whether behavioral signatures of visual attention might be unique to the human or primate brain. Yet, in recent years, studies have shown that many animals, from barn owl ${ }^{22}$, bluejays ${ }^{23}$, and archer fish ${ }^{24}$, can learn to utilize cues and context. Even fruit flies ${ }^{25}$ and bee ${ }^{26}$ with as little as 1 million neurons can learn to attend and manifest behavioral signatures that mimic those measured in humans. How can these simpler organisms learn such tasks from the visual input? Do their brains also implement these various attentional constructs from a serial spotlight or a limited resource? Are there simpler algorithms that give rise to the behavioral attention signatures without invoking more abstract attention concepts?

An alternative theoretical approach to classic attentional constructs posits that many (but not all ${ }^{27-31}$ ) behavioral attention effects can be explained by an organism's selection or weighted integration of multiple noise-limited sources of visual information ${ }^{3,32-38}$. Sensory neural signals are subject to statistical noise from the visual environment and/or internal to the brain's signal transduction ${ }^{39}$. Uncertainty about locations, features, and distractors degrades perceptual accuracy ${ }^{40,40-45}$. In this perspective, the cueing or set-size effects arise as a byproduct of optimally using all available information and excluding or down-weighting locations or distractors that are potentially confusable with the target ${ }^{3,33,46-51}$. This theoretical approach can be formalized by considering a mathematical 
model that makes inferences about the probabilities of each of the possible decision hypotheses given the sensory data and the prior probabilities that associate the context or cue to a target (ideal Bayesian observer $3,33,46-50$, Figure 2). Although the ideal Bayesian model (and Signal Detection approximations) have served as mathematically elegant benchmarks to compare against human performance $30,34,38,48,50,52-54$, they suffer various limitations ${ }^{55}$. The models have a priori all information about the cue and context, and often (but not always ${ }^{56}$ ) omit the process of learning its predictive probabilities, and importantly are more difficult to reconcile with a biologically plausible implementation (but see ${ }^{48,57}$ ). Thus, we do not know how a simple neural architecture with as little as a few million active neurons and $\sim 10^{7}$ synapses trained to detect targets in actual images automatically learns to utilize co-occurring cues or context. Previous work has shown how neural networks acting on a simplified stimulus ${ }^{8}$ can learn context or, acting on hypothetical Poisson neural responses ${ }^{58,59}$, learn to make probabilistic inferences. However, the approach has not been applied to full images and benchmarked against humans and image-computable ideal Bayesian observers for the three most common attention paradigms: Posner cueing, visual search with cues, and contextual cueing.

Here, we train a feedforward convolutional neural network with the same architecture on three classic attentional paradigms on actual images (Figure 2). The networks are given images with a label of whether a target is present or absent (Posner cueing and search tasks) or whether a right-tilted T or lefttilted $\mathrm{T}$ is present (contextual cueing). No information is given to the network about cues or context or their predictive probabilities. We assess whether the CNN shows the behavioral signatures that have defined human visual attention. We compare the CNNs overall performance against an imagecomputable ideal Bayesian observer ${ }^{60}$ that achieves the highest possible performance in the presence of a known source of noise added to the stimulus ${ }^{61}$. (See Supplementary Information S.1-6 for details of the Bayesian Ideal observer.) In each task, human observers maintained their gaze on a fixation point during a brief presentation of the display to isolate covert attention effects from the contributions of foveation through eye movements. 
Posner Paradigm

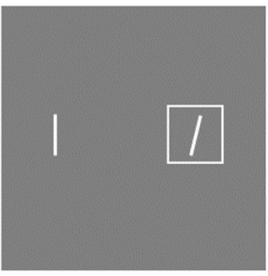

Stimulus with valid cue

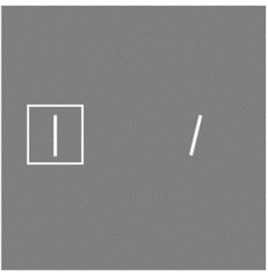

Stimulus with invalid cue
Visual Search

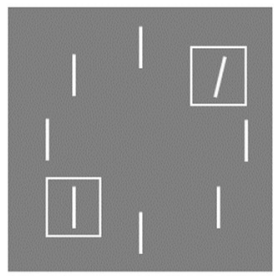

Set Size Two

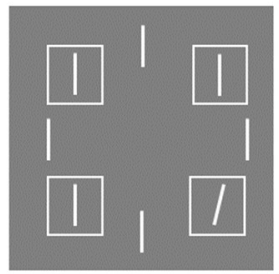

Set Size Four
Contextual Cueing

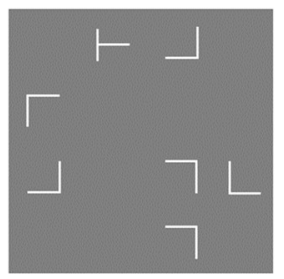

Old Configuration

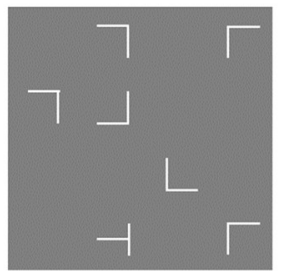

New Configuration

Figure 1. Examples of three classic experimental paradigms used to study and characterize human visual attention. Left column: In the Posner cueing paradigm, investigators compare performance for valid cue trials (the cue box correctly predicts the target - tilted line - location) relative to invalid cue trials (the cue box incorrectly predicts the target location). Middle column: In the visual search paradigm, investigators compare performance detecting the target (tilted line) as a function of the number of cues indicating likely target location (set-size). Right column: In the contextual cueing paradigm, investigators compare performance identifying the orientation of the target (left tilted $T$ vs. right tilted T) within a configuration that has been repeated (old configuration) or a new configuration. 
(a)
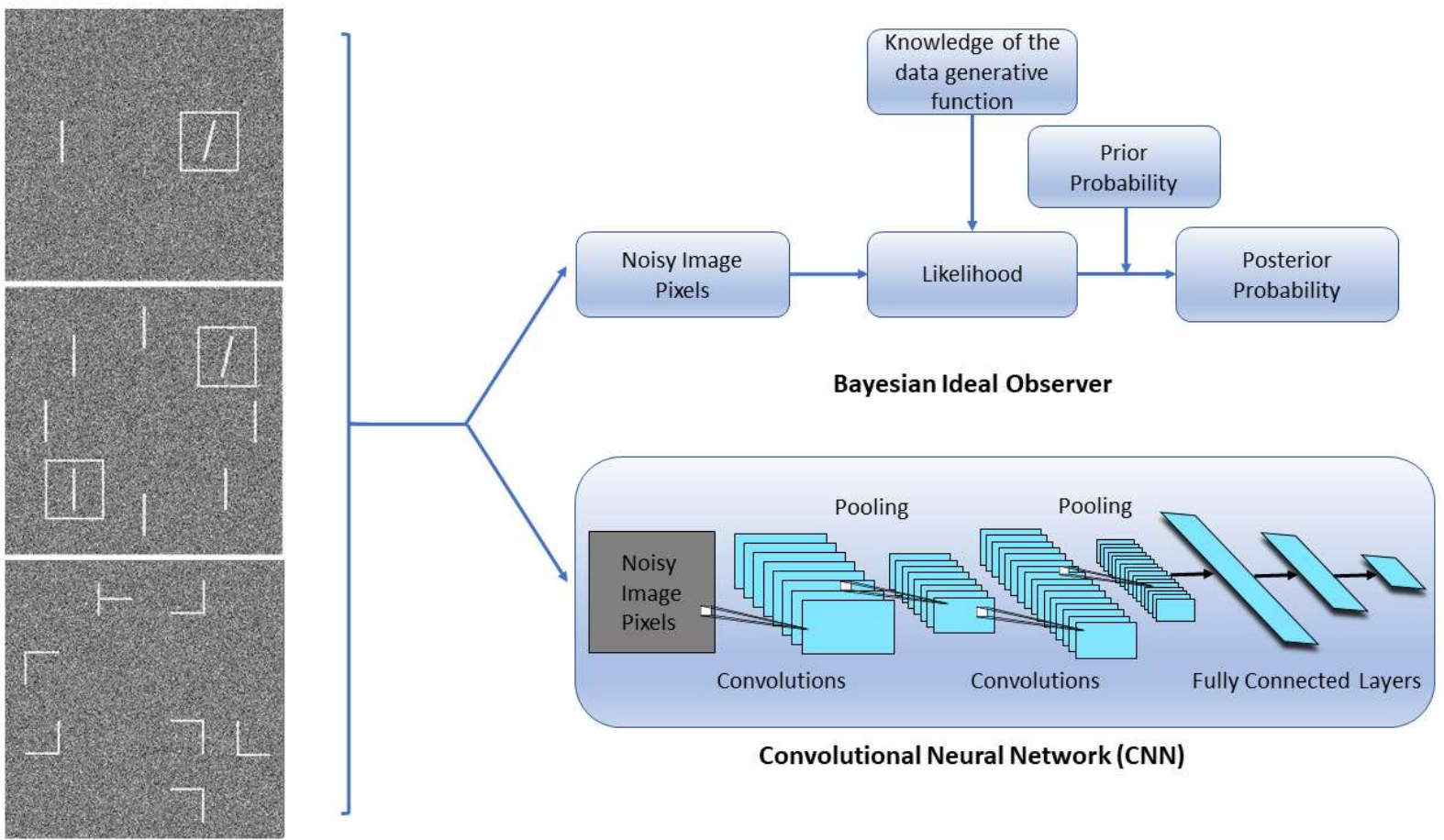

(b)

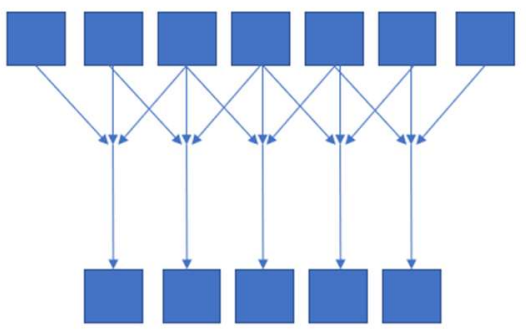

\author{
Input Pixels \\ One kernel which looks at three input \\ pixels and slides through the input \\ Output neurons integrating \\ input from multiple locations
}

Figure 2 (a): Left: Example stimuli used in the three experiments with external white noise (top: cueing paradigm; middle: visual search; bottom: contextual cueing). Right: Application of a Bayesian ideal observer (BIO) and Convolutional Neural Network (CNN). (b) A simple demonstration of how the number of neurons and synapses is defined for a convolution layer. There are seven input pixels, five output neurons, and 15 synapses (see Methods for more details). This convolution contributes five computations, and each output layer neuron receives the output of exactly one computation. Thus, the number of computations by a convolution layer is equal to the number of neurons in its output layer. Similarly, each output neuron receives inputs from three neurons in the input, and thus the number of synapses contributed by this layer is equal to the size of the output times the size of the kernel. 


\section{Results}

In the cueing paradigm, observers detected the presence of a tilted target line that appeared $50 \%$ of the trials. The target line was tilted at an angle sampled from a Gaussian distribution with a mean of 15 degrees and a standard deviation of 3.5 degrees. The remaining locations in the target-present trials contained a line tilted at an angle sampled from a distractor distribution with a mean of 7 degrees and a standard deviation of 3.5 degrees (Figure 1). In target-absent trials, the two locations contained lines with angles sampled from the distractor distribution. White noise (RMS $=0.33$ ) was added to the display. The orientations of all lines were perturbed with a Gaussian randomly distributed orientation noise independently added to each element on every trial. A box cue around one of the two elements was 80 $\%$ predictive of the target location (when present). Different conditions varied the contrast of the box cue to investigate the interactions between the box-cue visibility and the cueing effect. The CNN was a feedforward network with two convolution layers followed by two fully connected layers, 5.5 million neurons, and $10^{7}$ synapses (weight inputs, see Figure 2 for number neurons and synapses calculation). We first compared the overall performance of the human and the CNN to that of the BIO at the highest box cue contrast. The method involves finding the external white noise variance for the images that would result in a BIO performance that matches that human or the CNN perceptual accuracy. The ratio of the noise variance of the $\mathrm{BIO}$ to the $\mathrm{CNN}$ /human is used as a measure of relative performance and is known as the statistical efficiency ${ }^{61-65}$. A statistical efficiency (SE) of 1 indicates that the humans or a model perform as well as the $\mathrm{BIO}$. A ratio of 0.5 means that the $\mathrm{BIO}$ can afford double the noise variance to achieve the human or CNN performance. Figure 3 shows that the CNN approximates the BIO ( $\mathrm{SE}=90 \%$ ) for this task while humans achieve a statistical efficiency of $39 \%$. To further compare how humans behave across valid and invalid cue trials, we increased the external noise for the BIO and CNN to match the human results with two fitting parameters: the standard deviation of the external noise and a decision criterion to determine yes/no responses.

$$
\eta_{x}:=\frac{\text { variance of noise shown to } x}{\text { variance of noise shown to } I O}
$$

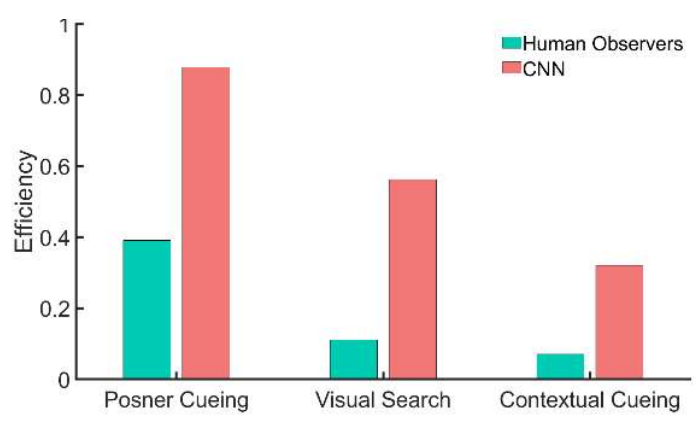

where $x$ denotes humans or CNN

Figure 3: Left: Definition of statistical efficiency to quantify the performance of humans and CNNs relative to that of the Ideal Observer (IO) by dividing the variance of the noise presented to the CNN or humans to match the 
performance of the IO) by the variance present in the images processed by the IO. Right: Efficiency of humans and CNN for the three tasks.

The first row of Table 1 shows the human data (averaged across six observers), CNN, and BIO predictions for the Posner cueing effect. Both the $\mathrm{CNN}$ and the BIO show a comparable cueing effect: higher hit rate for valid cue trials vs. invalid cue trials. As the contrast of the box cue is reduced, the cueing effect diminishes. However, the BIO that optimally detects the box results in a cueing effect even at the lowest (non-zero) box contrast. In contrast, the CNN and humans are suboptimal at detecting the cue box and require more box contrast to lead to a cueing effect. Overall, across all cue contrast conditions, the CNN provided a better fit to the data than the BIO (Akaike Information Criterion, AIC $=214.98$ for the $\mathrm{CNN}$ and $\mathrm{AIC}=200.65$ for the $\mathrm{BIO}$ ). Supplementary Information S.4 includes details of the computations used in the BIO for the Posner Cueing task.

Next, we compared the $\mathrm{BIO}$ and the CNN (with the same architecture as for the cueing paradigm) on a visual search task. The task was to correctly decide whether a target (tilted line) was present (50\% probability). Across trials, we manipulated the set size by varying the number of box cues $(n=1,2,4,8)$. The cue boxes predicted the likely location of the target when present. For target-present trials, the probability of the target being present at any cued location was $1 / n$. The target never appeared at the uncued locations. We first evaluated the statistical efficiency of the CNN and humans for the search task (Figure 3). The statistical efficiency for the CNN was $56.25 \%$, while $11.11 \%$ for the humans. We fit the BIO and CNN to the human data across set sizes with two fitting parameters. The external noise was adjusted so that the model's overall performance was similar to that of humans. The decision criterion, constrained to be the same across all set-sizes, was varied to best match the human hit and falsepositive rates. The $2^{\text {nd }}$ row of table 1 shows human, $\mathrm{BIO}$, and CNN results for the visual search task. Both the $\mathrm{BIO}$ and $\mathrm{CNN}$ capture the degradation in human perceptual performance with increasing set size. The $\mathrm{BIO}$ resulted in a better fit than the $\mathrm{CNN}$ across set-sizes $(\mathrm{AIC}=56.85$ for the $\mathrm{CNN}$ and $\mathrm{AIC}=$ 13.81 for the BIO). Supplementary Information S.5 includes details of the computations used in the BIO for the Visual Search task.

Finally, we compared the $\mathrm{CNN}$ and human performance in the contextual cueing paradigm to that of BIO. Here, observers decided whether the T was tilted right or left. In a first epoch (160 trials), human observers viewed the display for $1000 \mathrm{~ms}$ and were allowed to execute eye movements to familiarize themselves with the configurations. The remaining test sessions were conducted while observers maintained gaze on a fixation point and with 250 ms presentations. This allowed us to separate the effects of covert attention from eye movements ${ }^{66-68}$ on perceptual performance. There were five sessions of testing. We compare the BIO/CNN performance to human performance averaged over the $3^{\text {rd }}, 4^{\text {th }}$ and $5^{\text {th }}$ epochs where the contextual cueing stabilizes (see Methods and Materials for all sessions).

The BIO for the contextual cueing task should consider the likelihood of the image data given all possible background configurations. The combinatorial explosion of possible configurations $(656,015,360)$ makes the $\mathrm{BIO}$ to be computationally intractable. Thus, we approximated the BIO with a model that considered the old configurations and also evaluated element-wise information. This model can approximate the $\mathrm{BIO}$ (see Supplementary Information S.6 for details of the BIO, the BIO approximation, and the validation of the BIO approximation with a simpler display for which the exact BIO performance can be calculated). 
The architecture of the CNN evaluated remained the same as for the Posner cueing and search task. Figure 3 shows the statistical efficiency relative to the $\sim \mathrm{BIO}$ (the $\sim$ symbol denotes approximation) was lower than for the other tasks resulting in $7.15 \%$ for humans and $32.13 \%$ for the CNN. To compare relative performance across conditions, we increased the external noise for the $\sim \mathrm{BIO}$ and $\mathrm{CNN}$ to fit human performance. The third row of Table 1 shows $\mathrm{BIO}, \mathrm{CNN}$, and human performance. Both the BIO and CNN models predict the human advantage in identifying the orientation of the target with old configurations relative to new configurations ( $\mathrm{AIC}=8.16$ for the $\mathrm{CNN}$ and $\mathrm{AIC}=30.56$ for the $\mathrm{BIO}$ ). 
Ideal Observer and Humans

Posner Cueing

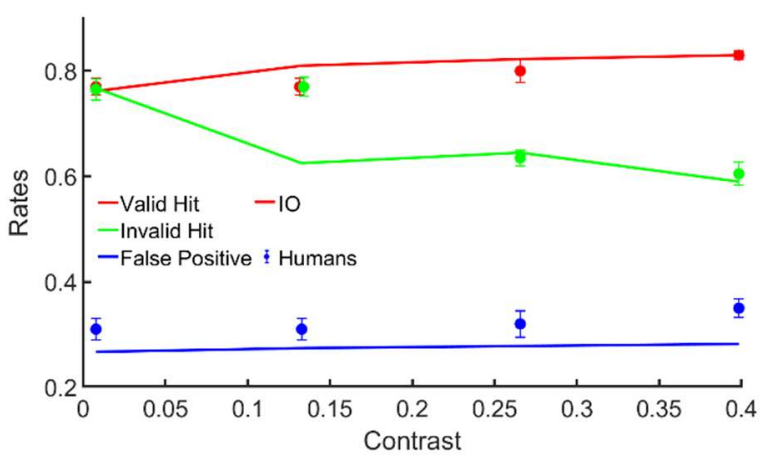

Visual

Search

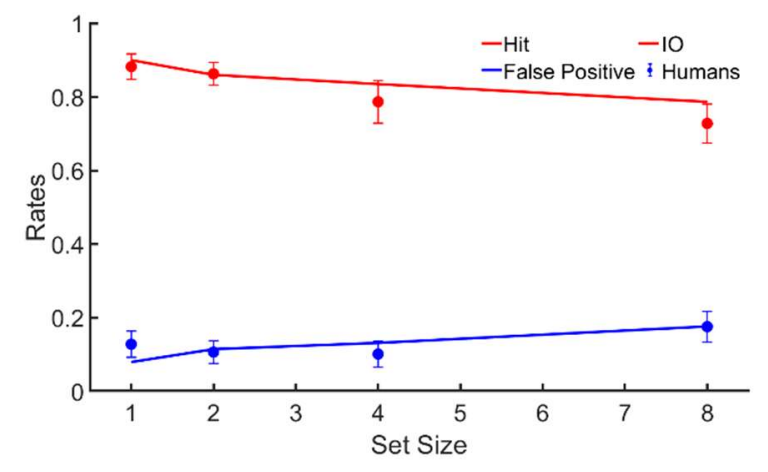

Contextual Cueing

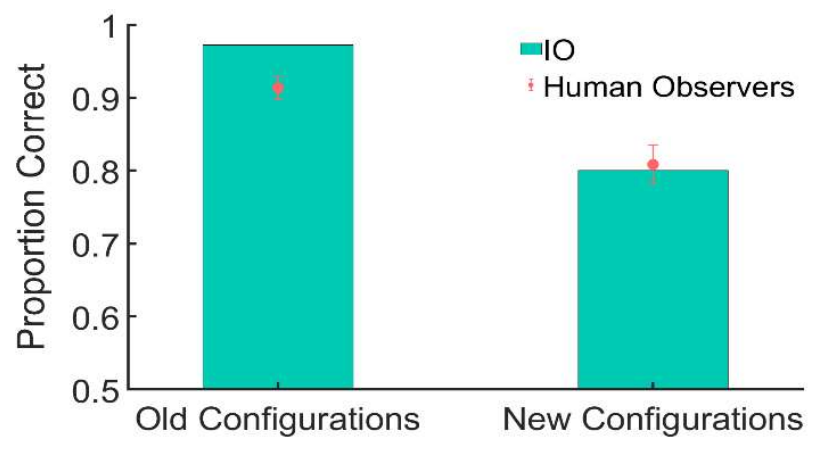

Convolution Neural Network and Humans
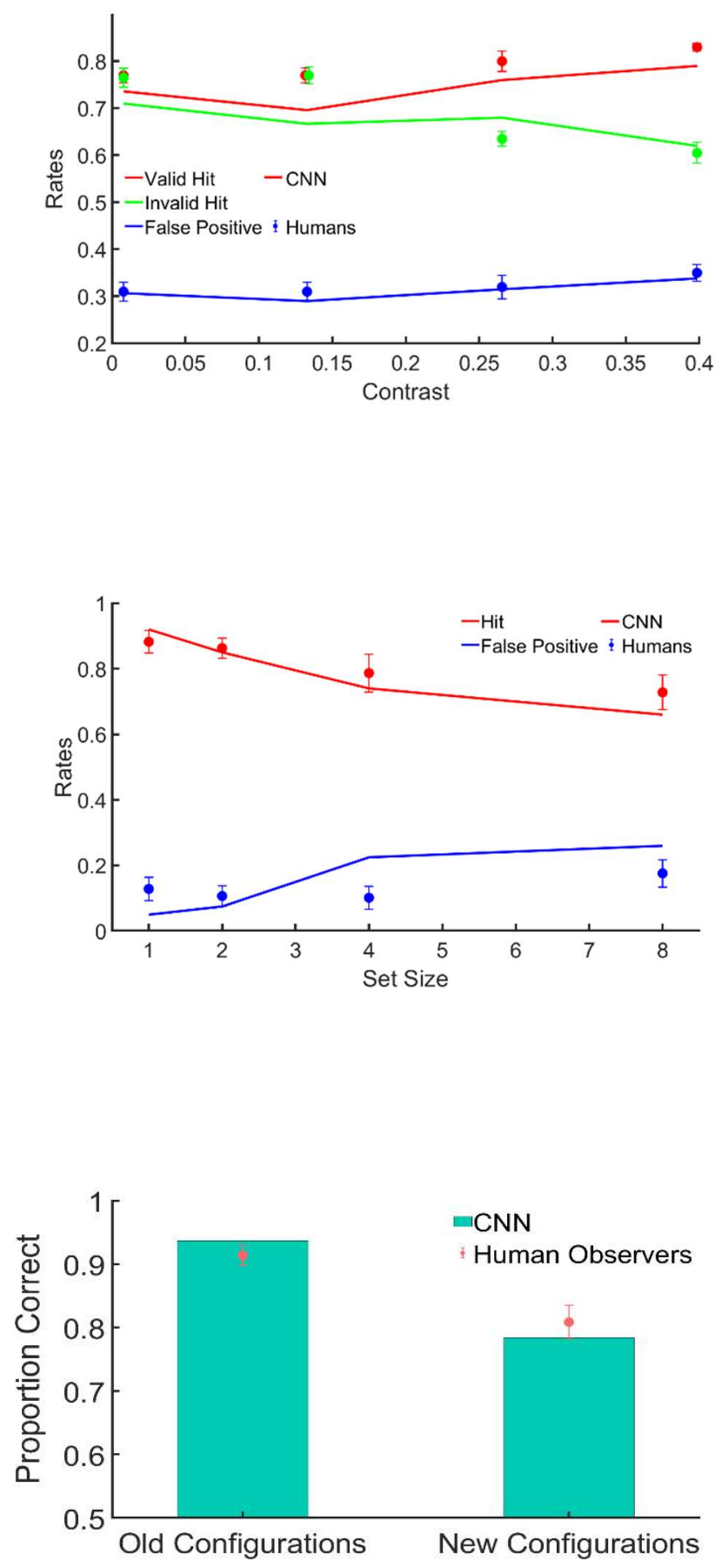
Table 1. Bayesian Ideal Observer (left column) and Convolution Neural Network (right column) comparison to human performance (data points with error bars) in the three classic attentional paradigms: Posner cueing paradigm, visual search, and contextual cueing. Errors denote the standard error of the mean across six human observers.

\section{Discussion}

We compared a CNN to the theoretical optimal achieved by an image-computable BIO and human observers of arguably the most common attention paradigms. The implementations of the $\mathrm{BIO}$ extend previous implementations $3,38,49,50,69$, which omitted the process of the detection of the cues and assumed it was detected without error. In addition, the current work introduces the BIO to the contextual cueing task by using a BIO approximation which is validated with a simple configuration.

In terms of absolute efficiency of the CNN relative to the BIO, the results showed the highest efficiency for simpler displays containing fewer elements (2-location cueing Posner task, $88 \%$ ) and lowest for the more complex display that contained more elements and element types ( $\sim 32 \%$ contextual cueing). Similarly, humans also achieved the highest absolute efficiency with simple displays and lowest with the contextual cueing display. In general, as the display included more variability of locations and possible element types (i.e., entropy), the CNN and humans tended to achieve lower statistical efficiency relative to the optimal observer.

The CNN predicted the benefits of the cues and context in all three paradigms. The CNN was better at predicting the Posner cueing effect as a function of cue contrast than the BIO. This is mostly because the BIO was more efficient at detecting the low-contrast box cue than the $\mathrm{CNN}$ and humans and thus over-predicted the cueing effect with the low contrast cue. Human and CNN performance illustrate how the cueing effect is modulated by the detectability of the cue, a fact that is seldom considered in the literature. For the contextual cueing task, the CNN was also better at predicting the human results than the BIO. For the visual search task, the BIO fit the human data somewhat better than the CNN that predicted smaller set-size effects.

The purpose of the work was not to determine which of the two models best predicted human performance but to illustrate how the CNN can automatically capture some of the cueing/context effects in humans, which are modeled in the BIO with known built-in prior probabilities. The implemented network included about 5.5 million neurons which is still higher than the approximately 1 million neurons of a bee ${ }^{70}$ or a few hundred thousand of a fruit fly ${ }^{71}$. Can similar results be obtained with a smaller CNN? Figure 4a shows that with over a 30-fold reduction in the network size to less than a quarter-million neurons (150K), the statistical efficiency relative to the BIO diminishes. And yet, the CNN can still learn the predictive cues and show cueing effects across all three experimental paradigms (Figure $4 \mathrm{~b}$ ) and achieve a higher performance than humans.

The current results contrast with previous findings showing that CNNs do not learn contextual relationships in more complex real scenes ${ }^{72}$. For example, humans use size relationships between objects and the surrounding scene to visual search, and human brain responses in scenes and attentionrelated areas are influenced by such associations ${ }^{73}$. Many $\mathrm{CNN}$-based object detectors are not sensitive to such object/scene size relationships ${ }^{72}$. Those results suggested that current CNNs might not have the 
capability to learn relationships among different elements in scenes. One possibility is that feedback (recurrent components to networks ${ }^{74,75}$ ), ubiquitous in the human brain ${ }^{75-78}$, is needed to learn relationships among elements in scenes. Feedback has been shown to improve object recognition ${ }^{79}$, learning long-range statistical dependencies ${ }^{74}$, and visual reasoning. Thus, feedback might be an important network property required to learn context and cues.
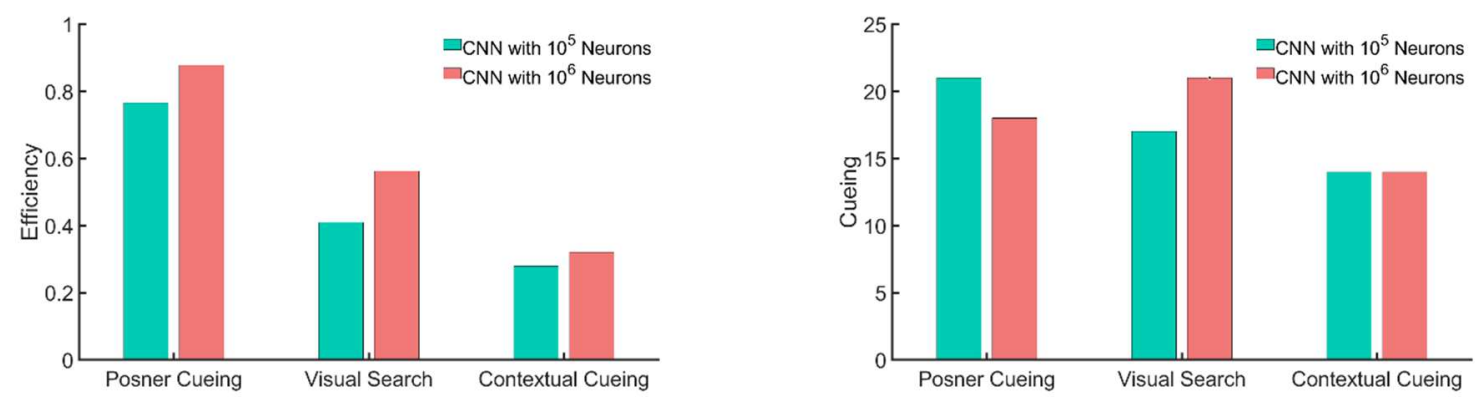

Figure 4: (a) Efficiencies and (b) cueing effect for the convolutional neural network after reducing the number of total neurons from $10^{6}$ to $10^{5}$ neurons. For the visual search task, the cueing effects were the difference between set-size 1 and 8.

The present findings show otherwise. At least for some of the simple classic tasks used to study visual attention, a simple feedforward network with less than a quarter-million neurons can learn to utilize the cues and results in cueing effects comparable to those measured in human observers.

There are various caveats to our interpretations. The feedforward network training involves backpropagation which adjusts the weight values by looking at the changes in the loss function at the output layer as a function of changes in weight in early layers. This process could be considered a form of feedback, although distinct from a dynamic feedback mechanism present in visual information processing at the testing stage. Another possible criticism of the current work is that the process of backpropagation might be non-biological ${ }^{80}$, although recent work has shown possible neural implementations that approximate backpropagation ${ }^{81-83}$. In the conventional backpropagation algorithm, changes in a neuron's weights can cause changes in the weights of neurons not directly connected, which disagrees with biology. If a predictive coding model is trained using only local Hebbian plasticity, the weight changes can converge to those in backpropagation ${ }^{81}$. Likewise, reinforcement learning can be implemented so that for a choice of action in the output layer, only those neurons in the previous layers which contribute to the action are modified ${ }^{83}$. This learning method is equivalent to backpropagation but for only one output unit at a time ${ }^{83}$, and its performance is comparable to standard backpropagation. Still, if brain networks do not have access to these learning processes that approximate backpropagation, the networks might need to rely on other forms of feedback mechanisms. 
To summarize, our results suggest that simple feedforward networks can learn and might represent a viable architecture that might explain how simpler organisms learn cues and contexts in laboratory experiments and how some ecological behaviors arise, such as blue jays selecting certain leaves more likely to contain a caterpillar ${ }^{84}$ or a bumblebee using flower types that are predictive of high rewards ${ }^{85}$.

Finally, feedback mechanisms are important in the primate brain ${ }^{76}, 79$, and identifying the tasks and learning that cannot be accomplished by simple feedforward networks is important to understand the unique functional contributions of feedback in human and animal visual cognition.

\section{References}

1. Posner, M. I. Orienting of attention. Q J Exp Psychol 32, 3-25 (1980).

2. Posner, M. I., Snyder, C. R. \& Davidson, B. J. Attention and the detection of signals. J Exp Psychol 109, 160-174 (1980).

3. Eckstein, M. P., Shimozaki, S. S. \& Abbey, C. K. The footprints of visual attention in the Posner cueing paradigm revealed by classification images. J Vis 2, 25-45 (2002).

4. Treisman, A. M. \& Gelade, G. A feature-integration theory of attention. Cogn Psychol 12, 97-136 (1980).

5. Treisman, A. Search, similarity, and integration of features between and within dimensions. J Exp Psychol Hum Percept Perform 17, 652-676 (1991).

6. Wolfe, J. M., Cave, K. R. \& Franzel, S. L. Guided search: an alternative to the feature integration model for visual search. J Exp Psychol Hum Percept Perform 15, 419-433 (1989).

7. Wolfe, J. M. Guided Search 2.0 A revised model of visual search. Psychonomic Bulletin \& Review 1, 202-238 (1994).

8. Brady, T. F. \& Chun, M. M. Spatial constraints on learning in visual search: modeling contextual cuing. J Exp Psychol Hum Percept Perform 33, 798-815 (2007).

9. Chun, M. M. Contextual cueing of visual attention. Trends Cogn. Sci. (Regul. Ed.) 4, 170-178 (2000). 
10. Kunar, M. A., Flusberg, S., Horowitz, T. S. \& Wolfe, J. M. Does contextual cuing guide the deployment of attention? Journal of Experimental Psychology: Human Perception and Performance 33,816 (2007).

11. Eriksen, C. W. \& St. James, J. D. Visual attention within and around the field of focal attention: A zoom lens model. Attention, Perception, \& Psychophysics 40, 225-240 (1986).

12. Luck, S. J., Hillyard, S. A., Mouloua, M. \& Hawkins, H. L. Mechanisms of visual-spatial attention: resource allocation or uncertainty reduction? J Exp Psychol Hum Percept Perform 22, 725-737 (1996).

13. Hawkins, H. L. et al. Visual attention modulates signal detectability. Journal of Experimental Psychology: Human Perception and Performance 16, 802-811 (1990).

14. Wolfe, J. M. Guided Search 4.0: Current Progress with a model of visual search. Integrated models of cognitive systems $25,1-57$ (2007).

15. Wolfe, J. M. Guided Search 6.0: An updated model of visual search. Psychon Bull Rev (2021) doi:10.3758/s13423-020-01859-9.

16. Brefczynski, J. A. \& DeYoe, E. A. A physiological correlate of the 'spotlight' of visual attention. Nat Neurosci 2, 370-374 (1999).

17. Chun, M. M. \& Jiang, Y. Contextual Cueing: Implicit Learning and Memory of Visual Context Guides Spatial Attention,. Cognitive Psychology 36, 28-71 (1998).

18. Chun, M. M. \& Jiang, Y. Top-Down Attentional Guidance Based on Implicit Learning of Visual Covariation. Psychological Science 10, 360-365 (1999).

19. Duncan, J. \& Humphreys, G. W. Visual search and stimulus similarity. Psychol Rev 96, 433-458 (1989).

20. Posner, M. Attentional Networks and Consciousness. Front. Psychol. 3, (2012). 
21. Tsuchiya, N. \& Boxtel, J. Introduction to research topic: Attention and consciousness in different senses. Frontiers in psychology 4, 249 (2013).

22. Lev-Ari, T., Zahar, Y., Agarwal, A. \& Gutfreund, Y. Behavioral and neuronal study of inhibition of return in barn owls. Scientific Reports 10, 7267 (2020).

23. Goto, K., Bond, A. B., Burks, M. \& Kamil, A. C. Visual Search and Attention in Blue Jays (Cyanocitta cristata): Associative Cuing and Sequential Priming. J Exp Psychol Anim Learn Cogn 40, 185-194 (2014).

24. Gabay, S., Leibovich, T., Ben-Simon, A., Henik, A. \& Segev, R. Inhibition of return in the archer fish. Nature Communications 4, 1657 (2013).

25. Sareen, P., Reinhard, W. \& Heisenberg, M. Attracting the attention of a fly | PNAS. https://www.pnas.org/content/108/17/7230.short.

26. Eckstein, M. P. et al. Rethinking human visual attention: spatial cueing effects and optimality of decisions by honeybees, monkeys and humans. Vision Res. 85, 5-19 (2013).

27. Dosher, B. A. \& Lu, Z. L. Mechanisms of perceptual attention in precuing of location. Vision research 40, 1269-1292 (2000).

28. Carrasco, M. Covert attention increases contrast sensitivity: Psychophysical, neurophysiological and neuroimaging studies. Prog. Brain Res 154, 33-70 (2006).

29. Carrasco, M. Visual attention: The past 25 years. Vision Research 51, 1484-1525 (2011).

30. Davis, E. T. et al. Mirror-image symmetry and search asymmetry: a comparison of their effects on visual search and a possible unifying explanation. Vision Res 46, 1263-1281 (2006).

31. Palmer, E. M., Fencsik, D. E., Flusberg, S. J., Horowitz, T. S. \& Wolfe, J. M. Signal detection evidence for limited capacity in visual search. Attention, Perception \& Psychophysics (2011) doi:10.3758/s13414-011-0199-2. 
32. Kinchla, R. A. Detecting target elements in multielement arrays: A confusability model. Attention, Perception, \& Psychophysics 15, 149-158 (1974).

33. Palmer, J. Set-size effects in visual search: the effect of attention is independent of the stimulus for simple tasks. Vision Res 34, 1703-1721 (1994).

34. Palmer, J. Attention in visual search: Distinguishing four causes of a set-size effect. Current Directions in Psychological Science 4, 118-123 (1995).

35. Shaw, M. L. Identifying attentional and decision making components in information processing. in In R.S. Nickerson (Ed.) Attention and performance VIII 277-296 (1980).

36. Sperling, G. \& Dosher, B. A. Strategy optimiation in human information processing. in Handbook of Perception and Human Performance (eds. Boff, K. R., Kaufman, L. \& Thomas, J. P.) vol. 1 2-1-2-65 (John Wiley and Sons, 1986).

37. Shimozaki, S. S., Eckstein, M. P. \& Abbey, C. K. Comparison of two weighted integration models for the cueing task: linear and likelihood. J Vis 3, 209-229 (2003).

38. Shimozaki, S. S., Schoonveld, W. A. \& Eckstein, M. P. A unified Bayesian observer analysis for set size and cueing effects on perceptual decisions and saccades. J Vis 12, (2012).

39. Tolhurst, D. J., Movshon, J. A. \& Dean, A. F. The statistical reliability of signals in single neurons in cat and monkey visual cortex. Vision Research 23, 775-785 (1983).

40. Pelli, D. G. Uncertainty explains many aspects of visual contrast detection and discrimination. J. Opt. Soc. Am. A 2, 1508-1531 (1985).

41. Cohn, T. E. \& Lasley, D. J. Detectability of a luminance increment: effect of spatial uncertainty. J Opt Soc Am 64, 1715-1719 (1974).

42. Davis, E. T., Kramer, P. \& Graham, N. Uncertainty about spatial frequency, spatial position, or contrast of visual patterns. Percept Psychophys 33, 20-28 (1983). 
43. Davis, E. T. \& Graham, N. Spatial frequency uncertainty effects in the detection of sinusoidal gratings. Vision Res 21, 705-712 (1981).

44. Burgess, A. E. \& Ghandeharian, H. Visual signal detection. II. Signal-location identification. J Opt Soc Am A 1, 906-910 (1984).

45. Bochud, F. O., Abbey, C. K. \& Eckstein, M. P. Search for lesions in mammograms: statistical characterization of observer responses. Med Phys 31, 24-36 (2004).

46. Eckstein, M. Probabilistic Computations for Attention, Eye Movements, and Search. Annu. Rev. Vis. Sci. 3:18.1-18.24, (2017).

47. Eckstein, M. P., Thomas, J. P., Palmer, J. \& Shimozaki, S. S. A signal detection model predicts the effects of set size on visual search accuracy for feature, conjunction, triple conjunction, and disjunction displays. Percept Psychophys 62, 425-451 (2000).

48. Ma, W. J., Navalpakkam, V., Beck, J. M., Berg, R. van den \& Pouget, A. Behavior and neural basis of near-optimal visual search. Nat. Neurosci 14, 783-790 (2011).

49. Vincent, B. T. Bayesian accounts of covert selective attention: A tutorial review. Atten Percept Psychophys 77, 1013-1032 (2015).

50. Vincent, B. T., Baddeley, R. J., Troscianko, T. \& Gilchrist, I. D. Optimal feature integration in visual search. J Vis 9, 15.1-11 (2009).

51. Pestilli, F., Carrasco, M., Heeger, D. J. \& Gardner, J. L. Attentional enhancement via selection and pooling of early sensory responses in human visual cortex. Neuron 72, 832-846 (2011).

52. Schoonveld, W., Shimozaki, S. S. \& Eckstein, M. P. Optimal observer model of single-fixation oddity search predicts a shallow set-size function. Journal of Vision 7, (2007).

53. Verghese, P. Visual search and attention: a signal detection theory approach. Neuron 31, 523-535 (2001).

54. Verghese, P. \& McKee, S. P. Visual search in clutter. Vision Res. 44, 1217-1225 (2004). 
55. Gardner, J. L. Optimality and heuristics in perceptual neuroscience. Nature Neuroscience 22, 514523 (2019).

56. Droll, J. A., Abbey, C. K. \& Eckstein, M. P. Learning cue validity through performance feedback. J Vis 9, 18.1-23 (2009).

57. Rao, R. P. N. Bayesian computation in recurrent neural circuits. Neural Comput 16, 1-38 (2004).

58. Quax, S. C., Bosch, S. E., Peelen, M. V. \& Gerven, M. A. J. van. Population codes of prior knowledge learned through environmental regularities. bioRxiv 688630 (2019) doi:10.1101/688630.

59. Orhan, A. E. \& Ma, W. J. Efficient probabilistic inference in generic neural networks trained with non-probabilistic feedback. Nature Communications 8, 138 (2017).

60. Geisler, W. S. Ideal Observer analysis. (MIT Press, 2003).

61. Burgess, A. E., Wagner, R. F., Jennings, R. J. \& Barlow, H. B. Efficiency of human visual signal discrimination. Science 214, 93-94 (1981).

62. Barlow, H. B. The absolute efficiency of perceptual decisions. Philos. Trans. R. Soc. Lond., B, Biol. Sci 290, 71-82 (1980).

63. Eckstein, M. P., Whiting, J. S. \& Thomas, J. P. Role of knowledge in human visual temporal integration in spatiotemporal noise. J Opt Soc Am A Opt Image Sci Vis 13, 1960-1968 (1996).

64. Tjan, B. S., Braje, W. L., Legge, G. E. \& Kersten, D. Human efficiency for recognizing 3-D objects in luminance noise. Vision Res 35, 3053-3069 (1995).

65. Pelli, D. G. \& Farell, B. Why use noise? J Opt Soc Am A Opt Image Sci Vis 16, 647-653 (1999).

66. Zelinsky, G. J. \& Sheinberg, D. L. Eye movements during parallel-serial visual search. J Exp Psychol Hum Percept Perform 23, 244-262 (1997).

67. Akbas, E. \& Eckstein, M. P. Object detection through search with a foveated visual system. PLOS Computational Biology 13, e1005743 (2017). 
68. Geisler, W. S. \& Chou, K.-L. Separation of low-level and high-level factors in complex tasks: Visual search. Psychological Review 102, 356-378 (1995).

69. Eckstein, M. P., Pham, B. T. \& Shimozaki, S. S. The footprints of visual attention during search with $100 \%$ valid and $100 \%$ invalid cues. Vision Res 44, 1193-1207 (2004).

70. Menzel, R. \& Giurfa, M. Cognitive architecture of a mini-brain: the honeybee. Trends in Cognitive Sciences 5, 62-71 (2001).

71. Raji, J. I. \& Potter, C. J. The number of neurons in Drosophila and mosquito brains. PLOS ONE 16, e0250381 (2021).

72. Eckstein, M. P., Koehler, K., Welbourne, L. E. \& Akbas, E. Humans, but Not Deep Neural Networks, Often Miss Giant Targets in Scenes. Current Biology 27, 2827-2832.e3 (2017).

73. Welbourne, L. E., Jonnalagadda, A., Giesbrecht, B. \& Eckstein, M. P. The transverse occipital sulcus and intraparietal sulcus show neural selectivity to object-scene size relationships. Commun Biol 4, 1-14 (2021).

74. Linsley, D., Kim, J., Veerabadran, V., Windolf, C. \& Serre, T. Learning long-range spatial dependencies with horizontal gated recurrent units. in Proceedings of the 32nd International Conference on Neural Information Processing Systems 152-164 (Curran Associates Inc., 2018).

75. Kreiman, G. \& Serre, T. Beyond the feedforward sweep: feedback computations in the visual cortex. Ann. N. Y. Acad. Sci. 1464, 222-241 (2020).

76. Lamme, V. A. \& Roelfsema, P. R. The distinct modes of vision offered by feedforward and recurrent processing. Trends Neurosci 23, 571-579 (2000).

77. Hasse, J. M. \& Briggs, F. Corticogeniculate feedback sharpens the temporal precision and spatial resolution of visual signals in the ferret. Proc Natl Acad Sci U S A 114, E6222-E6230 (2017).

78. Ling, S., Pratte, M. S. \& Tong, F. Attention alters orientation processing in the human lateral geniculate nucleus. Nature Neuroscience 18, 496-498 (2015). 
79. Kar, K., Kubilius, J., Schmidt, K., Issa, E. B. \& DiCarlo, J. J. Evidence that recurrent circuits are critical to the ventral stream's execution of core object recognition behavior. Nat Neurosci $22,974-983$ (2019).

80. Crick, F. The recent excitement about neural networks. Nature 337, 129-132 (1989).

81. Whittington, J. C. R. \& Bogacz, R. An Approximation of the Error Backpropagation Algorithm in a Predictive Coding Network with Local Hebbian Synaptic Plasticity. Neural Comput 29, 1229-1262 (2017).

82. Moldwin, T. \& Segev, I. Perceptron Learning and Classification in a Modeled Cortical Pyramidal Cell. Front. Comput. Neurosci. 14, (2020).

83. Pozzi, I., Bohté, S. M. \& Roelfsema, P. R. Attention-Gated Brain Propagation: How the brain can implement reward-based error backpropagation. 11.

84. Heinrich, B. \& Collins, S. L. Caterpillar Leaf Damage, and the Game of Hide-and-Seek with Birds. Ecology 64, 592-602 (1983).

85. Pleasants, J. M. Bumblebee Response to Variation in Nectar Availability. Ecology 62, 1648-1661 (1981). 


\section{Methods and Materials}

\section{Subjects}

Six undergraduate students from the University of California at Santa Barbara were recruited as subjects for the three experiments. The subjects all have a normal or corrected-to-normal vision. Four subjects were women, and two were men, all in the age range of 19-22. The subjects were naïve to the hypotheses of the study. The studies followed the human subject guidelines provided by the Institutional Review Board at the University of California, Santa Barbara.

\section{Eye Tracking and Experiment Setup}

The left eye of each participant was tracked using an SR Research Eyelink 1000 Tower Mount eye tracker sampling at $1000 \mathrm{~Hz}$. Before each 100-trial session, a 9-point calibration and validation were done, with a mean error of no more than 0.5 degrees of visual angle. In each experiment, participants were asked to fixate at a central cross. If they moved their eyes more than 1 degree from the fixation cross, an error message would appear, and the trial would abort and restart with a new stimulus.

\section{Stimuli and Psychophysics}

Stimuli for all experiments were generated using MATLAB. The mean pixel value of the image backgrounds was 128 . The minimum luminance was $0.08 \mathrm{~cd} / \mathrm{m}^{2}$ and the maximum $114.7 \mathrm{Cd} / \mathrm{m}^{2}$. The luminance was linear with greyscale. The display had a resolution of 1920 by 1080 pixels. The viewing distance was $75 \mathrm{~cm}$. The psychophysics experiment was written in MATLAB using the Psychophysics Toolbox.

\section{Posner Cueing Paradigm:}

We implemented a forced-fixation, yes-no version of the classic Posner Cueing Task (Posner, 1980) with varying levels of cue contrasts. Before each stimulus appeared on the screen, a blank screen with a fixation cross appeared on the screen for $250 \mathrm{~ms}$. Participants were asked to fixate at the fixation cross at the center of the screen. Next, the stimulus was presented on the screen for $250 \mathrm{~ms}$, with the fixation cross at the same location. An error message appeared if participants moved their fixation more than 1 degree away from the fixation cross, either on the pre-stimulus screen or while a stimulus was presented. When broken fixations were detected, the trial was restarted with a different stimulus. Following the stimulus presentation, a response screen was shown, asking participants to press the right arrow key if the target was present in the stimulus and the left arrow key otherwise. The response was recorded, and participants were asked to press the enter key to move on to the next stimulus. 


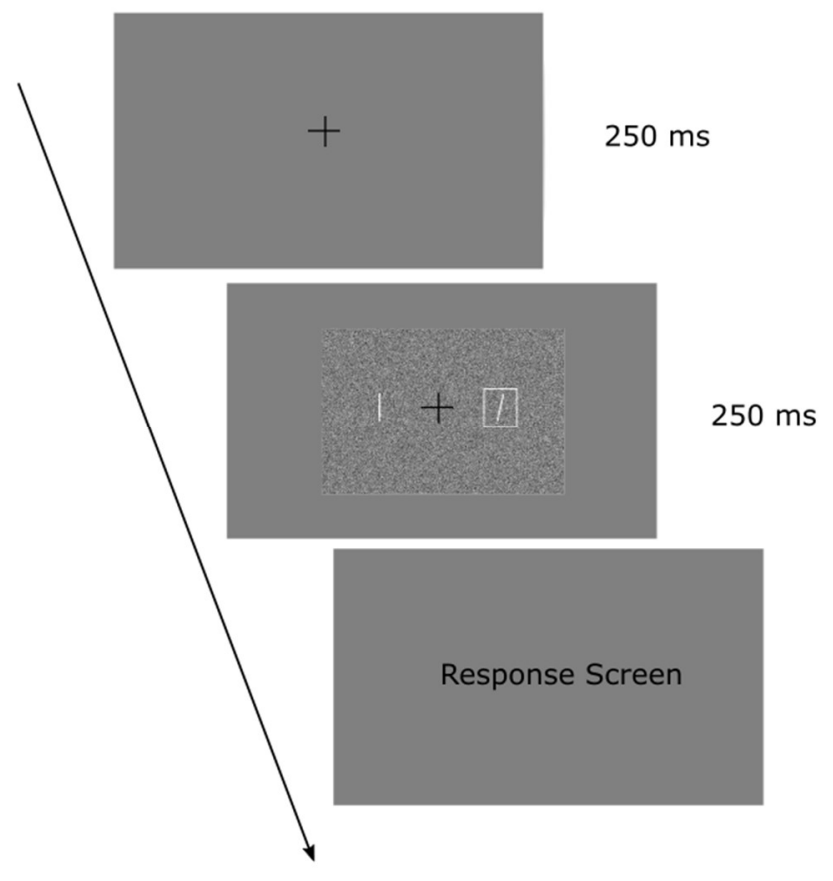

Figure 5: Experiment Sequence for Posner Cueing Task. Contrast of the target, distractor and box cue have been increased relative to the noise for display purposes.

Participants were shown 1600 stimuli in total over 16 blocks of 100 stimuli each, with each of the four box contrast levels being represented 400 times each. For each trial, each contrast level was equally likely to occur. Further, the stimulus contained a target with a $50 \%$ probability. When a target was present, it was present with the box with $80 \%$ probability and on the other side of the box with $20 \%$ probability. These trials are called valid cue trials and invalid cue trials, respectively. The box cue appeared on either side with equal probability. The target angle was sampled from a Gaussian distribution with a mean of 15 degrees and a standard deviation of 3.5 degrees. The distractor angle was sampled from a Gaussian distribution with a mean of 7 degrees and a standard deviation of 3.5 degrees. The noise contrast was 0.14 , while the target and the distractors had a contrast of 0.18 .

\section{Visual Search}

We implemented a forced fixation, yes-no visual search task with four different set sizes- 1, 2, 4, and 8 . Each image contained lines at 8 locations and boxes at varying number of locations, determined by the set size. All the set sizes were equally likely to appear. For set size 2 , only those configurations appear where the two boxes are diagonally opposite. For set size 4, there were two configurations, one with boxes at locations 1, 3, 5, and 7; and the other with boxes at locations 2, 4, 6, and 8. For each set size, each configuration was equally likely to appear.

For each trial, a blank screen with a fixation cross at the center appeared for 0.25 seconds, followed by the stimulus presentation for 0.25 seconds. An error message appeared if participants moved their fixation more than 0.5 degrees away from the fixation cross, either on the pre-stimulus screen or while a 
stimulus was presented, and the trial was restarted with a different stimulus. Following the stimulus presentation, a response screen was shown, asking participants to press the right arrow key if the target was present in the stimulus and the left arrow key otherwise. The response was recorded, and participants were asked to press the enter key to move on to the next stimulus. The noise contrast was 0.18 , while the target and the distractors had a contrast of 0.1 .

Participants were shown 1600 stimuli in total over 16 blocks of 100 stimuli each, with each set size being represented 400 times each. For each trial, each contrast level was equally likely to occur. Further, the stimulus contained a target with a $50 \%$ probability.

\section{Contextual Cueing}

This task was divided into 'epochs' consisting of five blocks each. Each block consisted of 32 trials. Within each block, 16 images are from a repeating set of configurations, referred to as old images. The other 16 images have configurations that are different for each block, and these are referred to as new images. The 16 old images have eight repeating backgrounds. Each background appears twice in each block, with the target appearing at the same location in both these images but with different orientations. For each block, the 16 new images, likewise, have the target in 8 configurations. Each of these new configurations appears twice in a block, with the target at the same locations in both images but oriented to the left in one image and the right in the other. The set of locations at which the target occurs in old images is disjoint with the set of locations at which the target occurs in new images. For each block, the target appears twice in each of the sixteen locations, once oriented to the left, the other time oriented to the right. The noise contrast was 0.33 , and the target, as well as the distractors, had a contrast of 0.5 .

The subjects were asked to maintain fixation at the center, and because of this, we also balanced the target eccentricities in the old and new configurations. Of the 16 locations in the image, the four corner locations have the highest eccentricities. Two of these corner locations were randomly chosen to occur with old configurations, and the other two with new configurations. The eight neighbors of the four corners have the next highest eccentricities. Four of these were randomly chosen to occur with old configurations, and the other four with new configurations. Finally, the four innermost locations all have the same eccentricities, and two of them were randomly chosen to occur with old configurations, the other two with new configurations.

In the first epoch (consisting of five blocks), the participants were allowed to make eye movements, and the stimulus was presented for 1 second. This was done to allow the participants to learn the association between the old configurations and the cued locations.

For the second to fifth epoch, participants were made to fixate at the center of the screen, and the stimuli were presented for 0.25 seconds.

For each epoch before the stimulus, a blank screen with a center cross appeared for 0.25 seconds. Following this, the stimulus is presented for 1 second (epoch 1 ) or 0.25 seconds (epoch 2 onward), with the center cross still intact. An error message appeared if participants moved their fixation more than 0.5 degrees away from the fixation cross, either on the pre-stimulus screen or while a stimulus was presented, and the trial was restarted with a different stimulus. Following the stimulus presentation, a 
response screen was shown, asking participants to press the right arrow key if the target was tilted to the right in the stimulus and the left arrow key otherwise. The response was recorded, and participants were asked to press the enter key to move on to the next stimulus.

\section{CNN Architecture}

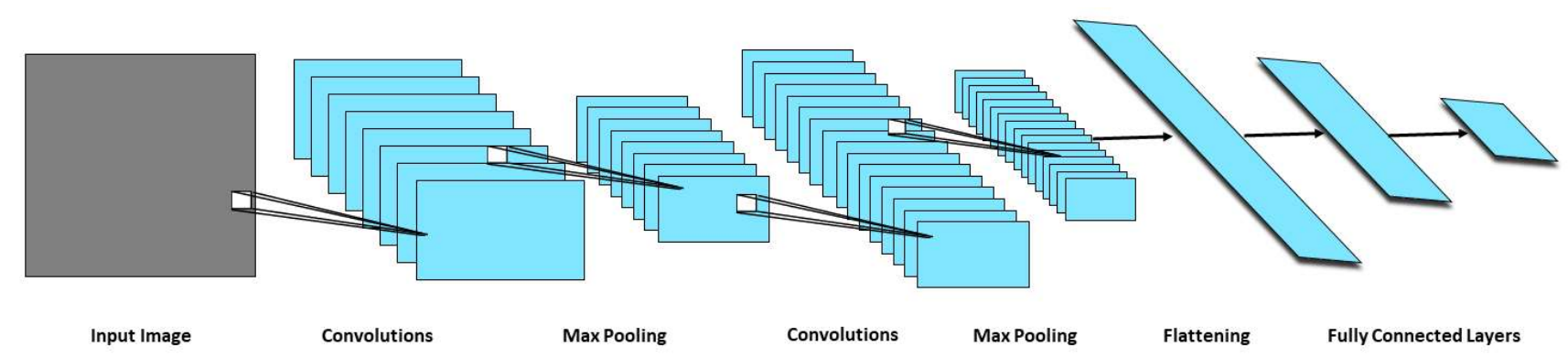

Figure 6: Flow Chart for CNN.

We used a feedforward convolutional neural network with two convolutional layers and two fully connected layers. ReLU activation function was used for all layers except the output layer where SoftMax activation was used.

The ReLU activation function is defined as:

$$
\operatorname{ReLU}(x)=\max (0, x)
$$

While the SoftMax function for a vector $\mathbf{z}=\left(z_{1}, z_{2}, \ldots, z_{k}\right)$ is defined as:

$$
\operatorname{SoftMax}(\mathbf{z})_{i}=\frac{e^{z_{i}}}{\sum_{j=1}^{K} e^{z_{j}}} \text { for } i=1,2, \ldots, K
$$

The training was done via gradient descent to minimize cross-entropy loss, with batch sizes of 32 , until convergence, which was typically around ten epochs.

The cross-entropy loss for CNN output $\hat{\boldsymbol{y}}$ and ground truth labels $\boldsymbol{y}$ for a batch of size 32 is defined as: 


$$
\sum_{n=1}^{32} y_{n 1} \log \hat{y}_{n 1}+\left(1-y_{n 2}\right) \log \left(1-\hat{y}_{n 2}\right)
$$

Where $\hat{y}_{n i}$ is the output of the $i^{\text {th }}$ neuron in the CNN to the $n^{\text {th }}$ image in the batch, and $y_{n i}$ is the $i^{\text {th }}$ component of the $n^{\text {th }}$ ground truth one-hot vector in the batch.

The proportion of various classes, conditions, and types of trials was the same as what human observers were shown. However, the number of images used for training and testing was higher. We used training and testing sizes of 10,000 images per set size for the visual search task, training size of 10,000 images and testing size of 10,000 images per box cue contrast for the Posner paradigm, and training and testing sample sizes of 10240 images for the contextual cueing task which corresponds to 160 epochs as defined in the human observer experiment. All images had independent samples of white Gaussian noise.

For the contextual cueing task, there were 16 old configurations corresponding to the 16 locations where the target could be placed. For each configuration, there was an image for each of the two target orientations totaling 32 images. Each of these 32 images was repeated 160 times in the training set, with an independent sample of white Gaussian noise being the only difference across multiple instances of the same images. Thus, there were a total of 5,120 old configuration images. The other half of the training set were new configuration images different from the old configuration images. In the new configuration images, the target was present on each location an equal number of times. Thus, each configuration appeared twice, once with the target pointing left, the other time with the target pointing right. The new images also had independent white Gaussian noise with the same mean and standard deviation as the old configuration images.

The test set for the CNN had the same 32 old configuration images appearing 5,120 times with independently sampled additive white Gaussian noise (different across samples and from the training image set). The new configuration images were another 5,120 images different from the configurations appearing in the training.

\section{Effective Number of Parameters used by the CNN}

We define the number of neurons in the network differently from the number of trainable parameters. While one convolution kernel only needs to learn one set of parameters that determines the kernel's weights, these are shared throughout the image it operates on. The number of computations (dot products) is a better metric to reflect the number of neurons in a brain. A simple demonstration for a 1dimensional input with a kernel of size three is shown in Figure 2(b). For a convolution layer or a pooling layer, the effective number of neurons it contributes is the number of units in its output. This is because each unit in the output of the convolution is the product of exactly one computation, and thus the number of computations done by a convolution layer is simply the size of its output. Likewise, we define the effective number of synapses. Each output unit receives input from one kernel, and thus, the number of synapses contributed by a convolution layer is equal to the size of the output times the kernel size. For a dense (fully connected) layer, the effective number of neurons it contributes is simply the number of neurons in the layer. Likewise, since for a dense layer, the input and output units are all 
connected, the number of synapses it contributes is simply the number of input units times the number of output units.

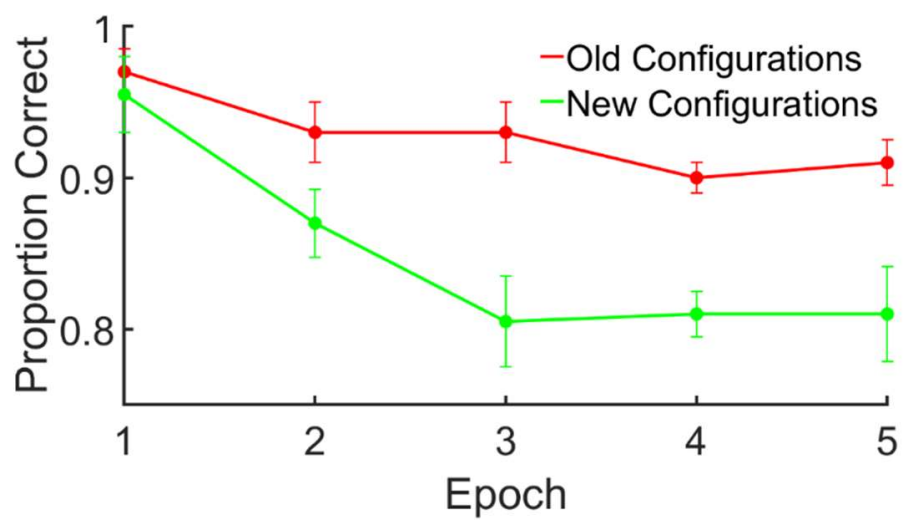

Figure 7: Human results for all epochs for the contextual cueing task. Errors denote the standard error of the mean across six human observers.

\section{Training a network whose size is comparable to the bee brain}

The CNNs discussed so far have more neurons than the brain of a honeybee by an order of magnitude. We thus trained a smaller neural network with roughly the same number of neurons with $10^{5}$ neurons by increasing the strides of all convolutions to 3 , reducing the number of convolutions in the first and second layers from 32 and 64 respectively to 8 and 32 respectively, and changing the factor of pooling in both pooling layers to 3 . 


\section{Supplementary Information}

\section{S.1. Bayesian Ideal Observer Models}

We describe the ideal observer models applied to the three tasks and their corresponding images. General developments of the Bayesian ideal observers can be found in many publications ${ }^{45,59,60,84-87}$. The current treatment differs from previous Bayesian ideal observer treatments for the Posner cueing task and visual search 3,39,49-51,53,68,88 in incorporating the process of the detection of the cue. Previous model treatments incorporate a prior reflecting the conditional probability of the presence of the target given the cue but implicitly assume the perfect detection of the cue. Here, we develop a theoretically more general model that explicitly calculates the likelihoods of the data given the joint presence of the cue and target, target alone, and cue alone. When the cue visibility is high, the model makes identical performance predictions to the BIO model that only uses the priors and assumes perfect detection of the cues. Novel to this paper is the approximation to a Bayesian ideal observer for the contextual cueing task.

In the BIO framework, a decision across hypotheses $s_{i}$ is arrived at by calculating a posterior probability of each hypothesis given the sensory data, $P\left(s_{i} \mid \boldsymbol{g}\right)^{59,89}$. Bayes' theorem relates the posterior probability of a hypothesis to the likelihood of the observed sensory data given the hypothesis, $P\left(\boldsymbol{g} \mid s_{i}\right)$ and the prior probability of the hypothesis, $P\left(s_{i}\right)$ :

$$
P\left(s_{i} \mid \boldsymbol{g}\right)=\frac{P\left(s_{i}\right) P\left(\boldsymbol{g} \mid s_{i}\right)}{P(\boldsymbol{g})}
$$

Typically, the denominator, $P(\boldsymbol{g})$, which is the probability of the data over all possible hypotheses, is a constant across the various hypotheses. Thus, the BIO chooses the hypothesis with the highest product of likelihood and prior.

\section{S.2. Calculating Likelihoods}

Throughout the following sections, we will make references to the likelihood terms defined here. Suppose we have an $\mathrm{n}$ by $\mathrm{m}$ image $(\boldsymbol{g})$ which contains either a luminance-defined element, a tilted line with orientation $1\left(s_{1}\right)$, or a tilted line with orientation $0\left(s_{0}\right)$ or a box with additive Gaussian independent noise (with mean $\mu$ and standard deviation $\sigma$ ). Thus, each pixel luminance, $g_{p}$ of $\boldsymbol{g}$ is a corresponding luminance pixel of $s_{i, p}$ (where the first subscript refers to the hypothesis and the second subscript the location in the image) and some additive noise.

Because the noise added is Gaussian, the likelihood of observing a pixel value $g_{p}$ given that the $\mathrm{i}^{\text {th }}$ signal is present is given by the normal probability density function: 


$$
P\left(g_{p} \mid s_{i, p}\right)=\frac{1}{\sqrt{2 \pi \sigma^{2}}} \exp \left(-\frac{\left(g_{p}-s_{i, p}\right)^{2}}{2 \sigma^{2}}\right)
$$

where $\sigma$ is the pixel standard deviation of the luminance noise. We note that the likelihoods are defined with respect to pixel luminance values rather than hypothetical internal responses, feature values, or neural responses.

Because the noise added to each pixel is independent and identical, the joint likelihood of all pixels given the presence of the $\mathrm{i}^{\text {th }}$ signal, $P\left(\boldsymbol{g} \mid s_{i}\right)$, reduces to the product of individual pixel likelihoods:

$$
\begin{array}{rl}
P\left(\boldsymbol{g} \mid s_{i}\right)=\prod_{p} & P\left(g_{p} \mid s_{i, p}\right)=\prod_{p} \frac{1}{\sqrt{2 \pi \sigma^{2}}} \exp \left(-\frac{\left(g_{p}-s_{i, p}\right)^{2}}{2 \sigma^{2}}\right) \\
& \propto \prod_{p} \exp \left(\frac{-g_{p}^{2}+2 g_{p} s_{i, p}-s_{i, p}^{2}}{2 \sigma^{2}}\right) \\
& \propto \exp \left(\frac{-\boldsymbol{g}^{T} \boldsymbol{g}+2 s_{i}^{T} \boldsymbol{g}-s_{i}^{T} s_{i}}{2 \sigma^{2}}\right)
\end{array}
$$

where $\boldsymbol{g}$ is a 1-D vectorized version of the image and $s_{i}$ is a vectorized version of the $\mathrm{i}^{\text {th }}$ signal. Eq (S.3) is a basic likelihood calculation and contains a template matching term $\left(s_{i}{ }^{T} \boldsymbol{g}\right)$. Variants of Eq. S.3 are used throughout the development of the BIO for each specific task.

\section{S.3. Combining likelihoods across within hypothesis sub-events}

Another important property that we will repeatedly in our development of the BIO for the various tasks is that of summing likelihoods. In basic probability, the probability of an event $A$ involves summing probabilities across all mutually exclusive events that qualify as $A: \quad P(A)=P\left(A_{1}\right)+P\left(A_{2}\right)+\ldots+P\left(A_{n}\right)$. Similarly, in many tasks calculating the likelihood of the data given the presence of the target involves considering different locations, orientations, etc., for the target and distractors. The likelihood is calculated for each of the possible events and then summed to obtain a sum of likelihoods of the data given the presence of the target.

$$
P\left(\boldsymbol{g} \mid s_{\boldsymbol{i}}\right)=\sum_{k=1}^{K} P\left(\boldsymbol{g} \mid s_{i, k}\right) \cdot \frac{P\left(s_{i, k}\right)}{P\left(s_{i}\right)}
$$

where $\frac{P\left(s_{i, k}\right)}{P\left(s_{i}\right)}$ is the prior probability of the $\mathrm{k}^{\text {th }}$ event or target parameter value. Evaluating Equation S.4 requires the BIO to know apriori all possible values or locations attained by the target and distractors.

In the next, sections we formulate the BIO for each of the specific tasks: Posner cueing, visual search, and contextual cueing. 


\section{S.4. Posner Paradigm}

In the Posner cueing task, the hypothesis $s^{+}$is that the target is present (target line with orientation 1 is at one location and a line with a smaller tilt, orientation 0 , is at a second location), and the hypothesis $s^{-}$is that the target is absent (both elements have lines with orientation 0 ). The box cue is present at either location with equal probability. The target appears with the cue with a probability of $80 \%$. The likelihood of the sensory data, $\boldsymbol{g}$, given the presence of the target has to be summed across the mutually exclusive locations of the target.

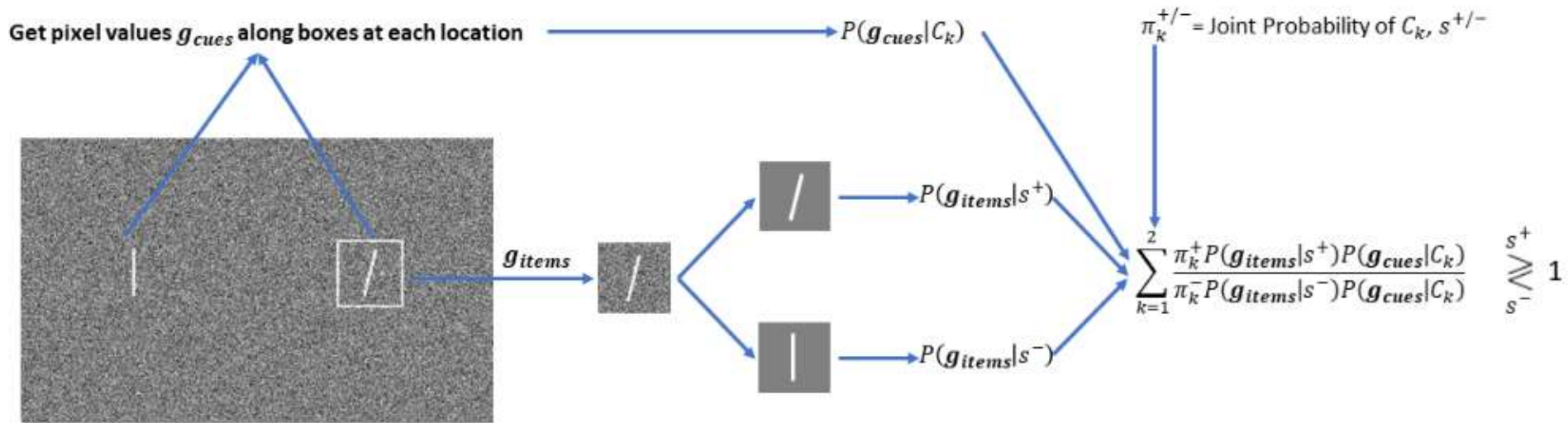

Get $g_{\text {items }}$ from each location

Template Matching

Supplementary Figure S.1: Flow Chart for the Ideal Observer for Posner Paradigm.

$$
P\left(s^{+} \mid \boldsymbol{g}\right)=\sum_{i=1}^{2} P\left(s_{i}^{+\prime} \mid \boldsymbol{g}\right)
$$

Where $s_{i}^{+\prime}$ stands for the target being present at location $\mathrm{i}$, and the target being absent at the other location.

And

$$
P\left(s_{1}^{+\prime} \mid \boldsymbol{g}\right)=P\left(s_{1}^{+} \mid \boldsymbol{g}_{\mathbf{1}}\right) \cdot P\left(s_{2}^{-} \mid \boldsymbol{g}_{\mathbf{2}}\right)
$$

$$
P\left(s_{2}^{+\prime} \mid \boldsymbol{g}\right)=P\left(s_{1}^{-} \mid \boldsymbol{g}_{\mathbf{1}}\right) \cdot P\left(s_{2}^{+} \mid \boldsymbol{g}_{\mathbf{2}}\right)
$$

where $g_{1}$ and $g_{2}$ are pixel values from the first and second locations, respectively. 
Marginalizing over the two configurations corresponding to the box cue appearing at the right or left location, we have

$$
P\left(s_{i}^{+\prime} \mid \boldsymbol{g}\right)=\sum_{k=1}^{2} P\left(s_{i}^{+\prime}, C_{K} \mid \boldsymbol{g}\right)
$$

Where $C_{1}$ refers to box on the Left Location, no box on the right location and $C_{2}$ refers to the box on the right location, no box on the left location.

Now,

$$
P\left(s_{i}^{+\prime}, C_{K} \mid \boldsymbol{g}\right) \propto P\left(\boldsymbol{g} \mid s_{i}^{+\prime}, C_{K}\right) \cdot P\left(C_{K}, s_{i}^{+\prime}\right)
$$

The likelihood can be separated into the independent contributions of the items (target/distractor) and the cues:

$$
P\left(\boldsymbol{g} \mid s_{i}^{+\prime}, C_{K}\right)=P\left(\boldsymbol{g}_{\text {items }}, \boldsymbol{g}_{\text {cues }} \mid s_{i}^{+\prime}, C_{K}\right)=P\left(\boldsymbol{g}_{\text {items }} \mid s_{i}^{+\prime}\right) . P\left(\boldsymbol{g}_{\text {cues }} \mid C_{K}\right)
$$

And

$$
P\left(C_{K}, s_{i}^{+\prime}\right)=P\left(s_{i}^{+\prime} \mid C_{K}\right) \cdot P\left(C_{K}\right)
$$

Thus, (S.9) reduces to:

$$
\begin{aligned}
P\left(s_{i}^{+\prime}, C_{K} \mid \boldsymbol{g}\right) \propto & P\left(\boldsymbol{g}_{\text {items }} \mid s_{i}^{+\prime}\right) \cdot P\left(\boldsymbol{g}_{\text {cues }} \mid C_{K}\right) \cdot P\left(s_{i}^{+\prime} \mid C_{K}\right) \cdot P\left(C_{K}\right) \\
& \propto P\left(\boldsymbol{g}_{\boldsymbol{i}} \mid s_{i}^{+}\right) \cdot P\left(\boldsymbol{g}_{! i} \mid s_{! i}^{-}\right) \cdot P\left(\boldsymbol{g}_{\text {cues }} \mid C_{K}\right) \cdot P\left(s_{i}^{+\prime} \mid C_{K}\right) \cdot P\left(C_{K}\right)
\end{aligned}
$$

Where $\boldsymbol{g}_{\boldsymbol{i}}$ indicates pixels from location i, and $\boldsymbol{g}_{! \boldsymbol{i}}$ indicates pixels from the location other than $\mathrm{i}$. $s_{i}^{+}$indicates target presence at location $\mathrm{i}$, while $s_{! i}^{-}$indicates distractor presence at the location other than $\mathrm{i}$.

Now, in our implementation of the Posner Paradigm, the target angle is sampled from a Gaussian distribution with a mean of 15 degrees and standard deviation of 3.5 degrees, while the distractor angle is sampled from a Gaussian distribution with a mean of 7 degrees and a standard deviation of 3.5 degrees. However, since the number of pixels is finite, only a finite number of angles can be drawn on the actual images of the target and distractors. While calculating the likelihood of a target being present, we marginalize across all possible angles along with their prior probabilities.

Thus, using equation (S.4), it follows that: 
And

$$
P\left(\boldsymbol{g}_{i} \mid s_{i}^{+}\right)=\sum_{\theta \in[0,2 \pi)} P\left(\boldsymbol{g}_{i} \mid s_{i \theta}^{+}\right) \cdot \frac{\pi_{\theta}^{+}}{P\left(s_{i}^{+}\right)}
$$

$$
P\left(\boldsymbol{g}_{! i} \mid s_{! i}^{-}\right)=\sum_{\theta \in[0,2 \pi)} P\left(\boldsymbol{g}_{! i} \mid s_{! i \theta}^{-}\right) \cdot \frac{\pi_{\theta}^{-}}{P\left(s_{! i}^{-}\right)}
$$

Where $s_{i \theta}^{+}$denotes the presence of the target with an angle $\theta$ at location i, $s_{! i \theta}^{-}$denotes the presence of the distractor with an angle $\theta$ at the location other than $\mathrm{i}, \pi_{\theta}^{+}$is the prior probability of sampling a template with angle $\theta$ from the target angle distribution and $\pi_{\theta}^{-}$is the prior probability of sampling a template with angle $\theta$ from the distractor angle distribution.

Thus, combining equations (S.5), (S.7), (S.9), and (S.12), we get:

$$
\begin{aligned}
& P\left(s^{+} \mid \boldsymbol{g}\right) \\
& \propto \sum_{i=1}^{2} \sum_{k=1}^{2} \sum_{\theta_{b} \in[0,2 \pi]} \sum_{\theta_{a} \in[0,2 \pi)} P\left(\boldsymbol{g}_{\boldsymbol{i}} \mid s_{i \theta_{a}}^{+}\right) P\left(\boldsymbol{g}_{! i} \mid s_{! i \theta_{b}}^{-}\right) \cdot P\left(\boldsymbol{g}_{\text {cues }} \mid C_{K}\right) \cdot P\left(s_{i}^{+\prime} \mid C_{K}\right) \cdot P\left(C_{K}\right) \cdot \frac{\pi_{\theta_{a}}^{+}}{P\left(s_{i}^{+}\right)} \cdot \frac{\pi_{\theta_{b}}^{-}}{P\left(s_{! i}^{-}\right)}
\end{aligned}
$$

The first three likelihood terms in the summand here are obtained using (eq (S.3)), and the last four terms are known. The four summations are across target location (i), cue location (k), target angle $(\theta$, and distractor angle $(\theta)$.

Likewise, to calculate the posterior probability for the target absence

$$
\begin{array}{r}
P\left(s^{-} \mid \boldsymbol{g}\right)=P\left(s_{1}^{-}, s_{2}^{-} \mid \boldsymbol{g}\right)=P\left(\boldsymbol{g} \mid s_{1}^{-}, s_{2}^{-}\right) \cdot \frac{P\left(s_{1}^{-}, s_{2}^{-}\right)}{P(\boldsymbol{g})} \propto 0.5 P\left(\boldsymbol{g} \mid s_{1}^{-}, s_{2}^{-}\right) \\
\propto 0.5 P\left(\boldsymbol{g}_{1}, \boldsymbol{g}_{2} \mid s_{1}^{-}, s_{2}^{-}\right) \propto 0.5 P\left(\boldsymbol{g}_{\mathbf{1}} \mid s_{1}^{-}\right) . P\left(\boldsymbol{g}_{2} \mid s_{2}^{-}\right)
\end{array}
$$

where $s_{i}^{-}$denotes the distractor being present at location i. $P\left(\boldsymbol{g}_{\mathbf{1}} \mid s_{1}^{-}\right)$and $P\left(\boldsymbol{g}_{2} \mid s_{2}^{-}\right)$are again marginalized over all possible distractor angles similar to (S.12).

Finally, the BIO makes the decision: target absent, $s^{-}$, if $P\left(s^{-} \mid \boldsymbol{g}\right)>P\left(s^{+} \mid \boldsymbol{g}\right)$ and target present, $s^{+}$, if $P\left(s^{-} \mid \boldsymbol{g}\right)<P\left(s^{+} \mid \boldsymbol{g}\right)$.

\section{S.5. Visual Search}

In the visual search task, the hypothesis $s^{+}$is that the target (tilted line) is present, and the hypothesis $s^{-}$is that the target is absent (all vertical lines). The target could appear at one of the eight locations. There were one, two, four, or eight box cues that could appear in M different configurations. The 
likelihood of the sensory data, $\boldsymbol{g}$, given the presence of the target has to be summed across the mutually exclusive locations of the target.

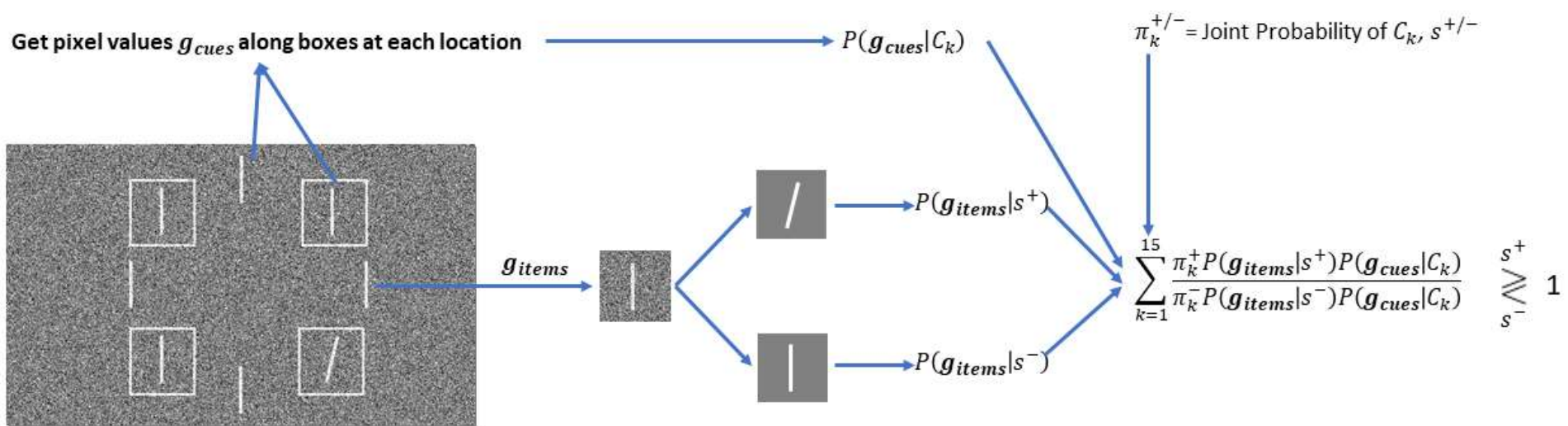

Get $g_{\text {items }}$ from each location

Template Matching

Supplementary Figure S.2: Flow Chart for the Ideal Observer for Visual Search.

$$
P\left(s^{+} \mid \boldsymbol{g}\right)=\sum_{i=1}^{8} P\left(s_{i}^{+\prime} \mid \boldsymbol{g}\right)
$$

Where $\boldsymbol{s}_{\boldsymbol{i}}^{+\prime}$ stands for the target being present at location $\mathrm{i}$, and the target being absent at all locations except location i.

In other words,

$$
P\left(s_{i}^{+\prime} \mid \boldsymbol{g}\right)=P\left(s_{i}^{+} \mid \boldsymbol{g}_{\boldsymbol{i}}\right) . \prod_{j !=i} P\left(s_{j}^{-} \mid \boldsymbol{g}_{\boldsymbol{j}}\right)
$$

Where $s_{i}^{+}$stands for the target being present at location $\mathrm{i}, s_{j}^{-}$stands for the distractor being present at location $\mathrm{j}$ and $\boldsymbol{g}_{\boldsymbol{i}}$ refers to pixel values from the location $\mathrm{j}$.

Because the box cue locations are randomized across trials, there are various mutually exclusive configurations that the likelihood needs to marginalize across;

$$
P\left(s_{i}^{+\prime} \mid \boldsymbol{g}\right)=\sum_{k=1}^{15} P\left(s_{i}^{+\prime}, C_{K} \mid \boldsymbol{g}\right)
$$


Where $C_{K}$ refers to Configuration k. Note that there were constraints on the box cue locations. There were evenly distributed across locations reducing the number of possible configurations ( 8 configurations for set-size one, 4 for set size two, 2 for set-size four, 1 for set size eight).

Now,

$$
P\left(s_{i}^{+\prime}, C_{K} \mid \boldsymbol{g}\right) \propto P\left(\boldsymbol{g} \mid s_{i}^{+\prime}, C_{k}\right) \cdot P\left(C_{K}, s_{i}^{+\prime}\right)
$$

We now separate the sensory data corresponding to the line items in the displays and the box cue.

$$
P\left(\boldsymbol{g} \mid s_{i}^{+\prime}, C_{K}\right)=P\left(\boldsymbol{g}_{\text {items }}, \boldsymbol{g}_{\text {cues }} \mid s_{i}^{+\prime}, C_{K}\right)=P\left(\boldsymbol{g}_{\text {items }} \mid s_{i}^{+\prime}\right) \cdot P\left(\boldsymbol{g}_{\text {cues }} \mid C_{K}\right)
$$

And

$$
P\left(C_{K}, s_{i}^{+\prime}\right)=P\left(s_{i}^{+\prime} \mid C_{K}\right) \cdot P\left(C_{K}\right)
$$

Thus, (S.18) reduces to:

$$
P\left(s_{i}^{+\prime}, C_{K} \mid \boldsymbol{g}\right) \propto P\left(\boldsymbol{g}_{\text {items }} \mid s_{i}^{+\prime}\right) \cdot P\left(\boldsymbol{g}_{\text {cues }} \mid C_{K}\right) \cdot P\left(s_{i}^{+\prime} \mid C_{K}\right) \cdot P\left(C_{K}\right)
$$

The first two terms here are obtained using the likelihood calculated in Eq (S.3), and the last two terms are known.

Likewise, we calculate $P\left(s^{-} \mid \boldsymbol{g}\right)$ as follows:

$$
\begin{aligned}
P\left(s^{-} \mid \boldsymbol{g}\right)=P\left(s_{1}^{-}, s_{2}^{-}, \ldots, s_{8}^{-} \mid \boldsymbol{g}\right)=\sum_{k=1}^{15} P\left(s_{1}^{-}, s_{2}^{-}, \ldots, s_{8}^{-}, C_{K} \mid \boldsymbol{g}\right) \\
\propto \sum_{k=1}^{15} P\left(\boldsymbol{g}_{\text {items }} \mid s_{1}^{-}, s_{2}^{-}, \ldots, s_{8}^{-}\right) \cdot P\left(\boldsymbol{g}_{\text {cues }} \mid C_{k}\right) \cdot P\left(s_{1}^{-}, s_{2}^{-}, \ldots, s_{8}^{-} \mid C_{k}\right) \cdot P\left(C_{k}\right)
\end{aligned}
$$

Where $s_{i}^{-}$denotes the distractor being present at location $\mathrm{i}$. 
Finally, we decide $s^{-}$if $P\left(s^{-} \mid \boldsymbol{g}\right)>P\left(s^{+} \mid \boldsymbol{g}\right)$ and $s^{+}$if $P\left(s^{-} \mid \boldsymbol{g}\right)<P\left(s^{+} \mid \boldsymbol{g}\right)$.

\section{S.6. Contextual Cueing}

For contextual cueing, there are two hypotheses. The hypothesis $S_{R}$ is that the rightward tilted $T$ is present, and the hypothesis $\mathrm{s}_{\mathrm{L}}$ is that the leftward tilted $\mathrm{T}$ is present. Calculating the likelihood of the sensory data given the presence of either of the targets involves marginalizing across all possible target locations and background configurations.

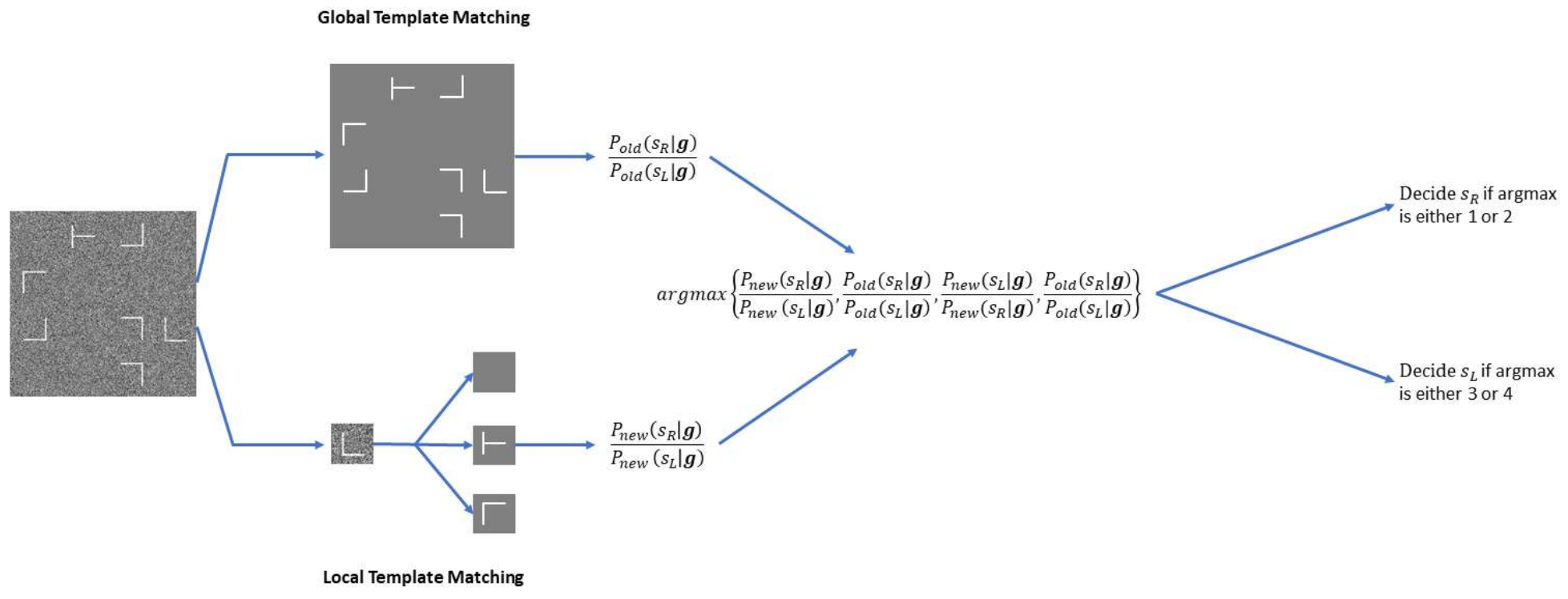

Supplementary Figure S.3: Flow Chart for approximation to the Bayesian Ideal Observer for Contextual Cueing

Ideally, for the likelihood, the observer should go through all possible configurations and assign a higher prior to old configurations ( $0.5 /$ number of old configurations) and a lower prior to new configurations (0.5/number of new configurations).

The BIO thus calculates

$$
P\left(s_{R} \mid \boldsymbol{g}\right)=\sum_{i=1}^{N_{\text {old }}} P\left(C_{O, R, i} \mid \boldsymbol{g}\right)+\sum_{i=1}^{N_{\text {new }}} P\left(C_{N, R, i} \mid \boldsymbol{g}\right)
$$

And 


$$
P\left(s_{L} \mid \boldsymbol{g}\right)=\sum_{i=1}^{N_{\text {old }}} P\left(C_{O, L, i} \mid \boldsymbol{g}\right)+\sum_{i=1}^{N_{\text {new }}} P\left(C_{N, L, i} \mid \boldsymbol{g}\right)
$$

Where $C_{O, R, i}$ denotes the $i^{\text {th }}$ old configuration with the rightward tilted target, $C_{N, R, i}$ denotes the $i^{\text {th }}$ new configuration with the rightward tilted target, $C_{O, L, i}$ denotes the $i^{\text {th }}$ old configuration with the leftward tilted target, $C_{N, L, i}$ denotes the $i^{\text {th }}$ old configuration with the leftward tilted target and $N_{\text {new }}$ and $N_{\text {old }}$ denote the total number of new and old configurations with a given target orientation, respectively.

Now, using Bayes Theorem,

$$
\begin{gathered}
P\left(C_{O, R, i} \mid \boldsymbol{g}\right)=P\left(\boldsymbol{g} \mid C_{O, R, i}\right) \cdot \frac{0.5}{N_{\text {old }}} ; \\
P\left(C_{O, L, i} \mid \boldsymbol{g}\right)=P\left(\boldsymbol{g} \mid C_{O, L, i}\right) \cdot \frac{0.5}{N_{\text {old }}} ; \\
P\left(C_{N, R, i} \mid \boldsymbol{g}\right)=P\left(\boldsymbol{g} \mid C_{N, R, i}\right) \cdot \frac{0.5}{N_{\text {new }}} ; \\
P\left(C_{N, L, i} \mid \boldsymbol{g}\right)=P\left(\boldsymbol{g} \mid C_{N, L, i}\right) \cdot \frac{0.5}{N_{\text {new }}}
\end{gathered}
$$

Now, each of $P\left(\boldsymbol{g} \mid C_{O, R, i}\right), P\left(\boldsymbol{g} \mid C_{O, L, i}\right), P\left(\boldsymbol{g} \mid C_{N, R, i}\right)$, and $P\left(\boldsymbol{g} \mid C_{N, L, i}\right)$ can be calculated using equation (S.3).

For our display with six Ls elements with four possible orientations, one T, nine blank locations in a $4 \times 4$ element array, and 16 possible target locations, however, the BIO requires calculating a summation across over 650 million configurations $(656,015,360)$. Such calculation is computationally intractable. Thus, we developed a model that approximates the BIO ( BIO) but is computationally more tractable. We first present the $\sim \mathrm{BIO}$ model and then validate it using a display with fewer elements for which the $\mathrm{BIO}$ and $\sim \mathrm{BIO}$ model performance can be calculated.

The $\sim \mathrm{BIO}$ consists of an optimal model that uses target and background information for the old configurations and a model that uses only local image patches. Thus, this suboptimal model differs from the $\mathrm{BIO}$ in that it does not use background information about all possible new configurations that could appear. In a sense, the $\sim \mathrm{BIO}$ might be a better model for humans. It is unlikely that human observers consider all possible new configurations that might appear.

The local IO has templates of both target types and all distractor types (4 orientations of Ls and one template for blank (no T or L). 
The local 10 gets the data $\boldsymbol{g}_{\boldsymbol{j}}$ from each location j and returns $P\left(\boldsymbol{s}_{\boldsymbol{R}} \mid \boldsymbol{g}\right)$ and $P\left(\boldsymbol{s}_{\boldsymbol{L}} \mid \boldsymbol{g}\right)$ where $\boldsymbol{s}_{\boldsymbol{R}}$ and $\boldsymbol{s}_{\boldsymbol{L}}$ refer to the target being oriented to the right and to the left, respectively.

We have:

$$
P\left(s_{R} \mid \boldsymbol{g}\right)=\sum_{j=1}^{16} P\left(s_{R j} \mid \boldsymbol{g}_{\boldsymbol{j}}\right) \cdot \prod_{i !=j} P\left(s_{D i} \mid \boldsymbol{g}_{\boldsymbol{i}}\right)
$$

Where $P\left(\boldsymbol{s}_{\boldsymbol{D} \boldsymbol{i}} \mid G_{i}\right)$ denotes the presence of a distractor at location i.

Clearly,

$$
\prod_{i !=j} P\left(s_{D i} \mid \boldsymbol{g}_{\boldsymbol{i}}\right)=\frac{\prod_{i=1}^{16} P\left(s_{D i} \mid \boldsymbol{g}_{\boldsymbol{i}}\right)}{P\left(s_{D j} \mid \boldsymbol{g}_{\boldsymbol{j}}\right)}
$$

Notice that $\prod_{i=1}^{16} P\left(s_{D i} \mid \boldsymbol{g}_{\boldsymbol{i}}\right)$ does not change on changing i, so let $k:=\prod_{i=1}^{16} P\left(s_{D i} \mid \boldsymbol{g}_{\boldsymbol{i}}\right)$.

Thus,

$$
P\left(s_{R} \mid \boldsymbol{g}\right)=k \cdot \sum_{j=1}^{16} \frac{P\left(s_{R j} \mid \boldsymbol{g}_{\boldsymbol{j}}\right)}{P\left(s_{D j} \mid \boldsymbol{g}_{\boldsymbol{j}}\right)}
$$

Likewise,

$$
P\left(s_{L} \mid \boldsymbol{g}\right)=k \cdot \sum_{j=1}^{16} \frac{P\left(s_{L j} \mid \boldsymbol{g}_{\boldsymbol{j}}\right)}{P\left(s_{D j} \mid \boldsymbol{g}_{\boldsymbol{j}}\right)}
$$

Therefore, k cancels out while calculating the likelihood ratio, and thus we need not calculate $k$.

$$
P\left(s_{R j} \mid \boldsymbol{g}_{\boldsymbol{j}}\right)=P\left(\boldsymbol{g}_{\boldsymbol{j}} \mid s_{R j}\right) \cdot P\left(s_{R j}\right) / P\left(\boldsymbol{g}_{\boldsymbol{j}}\right)
$$




$$
P\left(s_{D j} \mid \boldsymbol{g}_{\boldsymbol{j}}\right)=P\left(\boldsymbol{g}_{\boldsymbol{j}} \mid s_{D j}\right) \cdot P\left(s_{D j}\right) / P\left(\boldsymbol{g}_{\boldsymbol{j}}\right)
$$

$P\left(\boldsymbol{g}_{\boldsymbol{j}} \mid s_{R j}\right)$ and $P\left(\boldsymbol{g}_{\boldsymbol{j}} \mid s_{D j}\right)$ can be calculated via template matching (eq (S.3)), $P\left(s_{R j}\right)$ and $P\left(s_{D j}\right)$ is known and $P\left(\boldsymbol{g}_{\boldsymbol{j}}\right)$ cancels out.

Global IO: The global IO has 32 templates; 16 of them have the target pointing to the left and another 16 pointing to the right. There are 16 backgrounds, each background appearing twice, with one containing the target oriented to the left, the other to the right. For a given background, the target appears at the same location for both orientations.

$$
\begin{aligned}
& P\left(s_{R} \mid \boldsymbol{g}\right)=\sum_{i=1}^{16} P\left(s_{T R i} \mid \boldsymbol{g}\right) \\
& P\left(s_{L} \mid \boldsymbol{g}\right)=\sum_{i=1}^{16} P\left(s_{T L i} \mid \boldsymbol{g}\right)
\end{aligned}
$$

Where $P\left(s_{T R i} \mid \boldsymbol{I}\right)$ denotes the probability that the $i^{\text {th }}$ template with target pointing right is present in the image and $P\left(s_{T L i} \mid \boldsymbol{I}\right)$ denotes the probability that the $i^{\text {th }}$ template with target pointing left is present in the image.

$$
P\left(s_{T R i} \mid \boldsymbol{g}\right)=P\left(\boldsymbol{g} \mid s_{T R i}\right) \cdot P\left(s_{T R i}\right) / P(\boldsymbol{g})
$$

$P\left(\boldsymbol{g} \mid s_{T R i}\right)$ is calculated via template matching (eq(S.3)) and $P\left(s_{T R i}\right)=1 / 32$ and $P(\boldsymbol{g})$ cancels out while taking the likelihood ratio.

\section{Integrating the two models:}

Let $P_{\text {new }}$ denote the likelihood returned by the local IO, and $P_{\text {old }}$ denote the likelihood returned by the global IO. We calculate:

$$
\operatorname{argmax}\left\{\frac{P_{\text {new }}\left(s_{R} \mid \boldsymbol{g}\right)}{P_{\text {new }}\left(s_{L} \mid \boldsymbol{g}\right)}, \frac{P_{\text {old }}\left(s_{R} \mid \boldsymbol{g}\right)}{P_{\text {old }}\left(s_{L} \mid \boldsymbol{g}\right)}, \frac{P_{\text {new }}\left(s_{L} \mid \boldsymbol{g}\right)}{P_{\text {new }}\left(s_{R} \mid \boldsymbol{g}\right)}, \frac{P_{\text {old }}\left(s_{R} \mid \boldsymbol{g}\right)}{P_{\text {old }}\left(s_{L} \mid \boldsymbol{g}\right)}\right\}
$$

If the argmax returns 1 or 2 , the integrated model decides that the target is oriented to the right; else, if the argmax returns 3 or 4 , the integrated model decides that the target is oriented to the left. 


\section{Validation of approximation to the Bayesian ideal bserver with a smaller contextual cueing image set}

We have used an approximate version of the ideal observer for contextual cueing because it is not feasible to run the ideal observer because of the large number of possible configurations. To validate that the $\sim \mathrm{BIO}$ is a good approximation of the actual BIO, we created a scenario that included fewer configurations and for which both model performances could be calculated and compared. We made smaller images with only 4 locations, containing one target, two Ls, and one blank. Further, we reduced the number of possible orientations of $L$ from 4 to 2 . This results in a total of 96 possible configurations, 8 out of which are old configurations and 88 are new.

We ran the ideal observer on this set of images as well as our approximate ideal observer ( BIO). The $\sim \mathrm{BIO}$ obtained an efficiency of $\mathbf{9 3 \%}$ relative to the ideal observer and a contextual cueing (proportion correct for old configurations - proportion correct for new configurations) of 3.2, as compared to a contextual cueing of 2.9 for BIO.

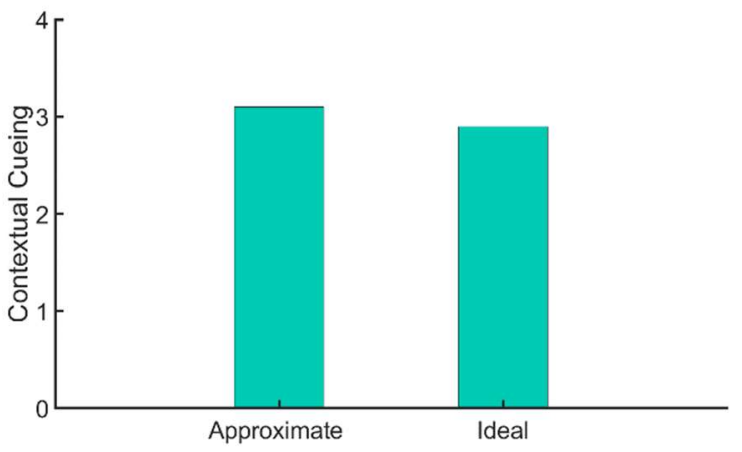

Supplementary Figure S.4: Contextual Cueing (Proportion correct for old configurations - Proportion correct for new configurations). Performance for the approximation to the ideal observer ( $\sim \mathrm{BIO})$ vs. the actual Bayesian ideal observer 